\title{
Tumor-Recruited Neutrophils and Neutrophil TIMP-Free MMP-9 Regulate Coordinately the Levels of Tumor Angiogenesis and Efficiency of Malignant Cell Intravasation
}

Erin M. Bekes, Bernhard Schweighofer, Tatyana A. Kupriyanova, Ewa Zajac, Veronica C. Ardi, James P. Quigley, and Elena I. Deryugina

From the Department of Cell Biology, The Scripps Research Institute, La Jolla, California

Tumor-associated neutrophils contribute to neovascularization by supplying matrix metalloproteinase-9 (MMP-9), a protease that has been genetically and biochemically linked to induction of angiogenesis. Specific roles of inflammatory neutrophils and their distinct proMMP-9 in the coordinate regulation of tumor angiogenesis and tumor cell dissemination, however, have not been addressed. We demonstrate that the primary tumors formed by highly disseminating variants of human fibrosarcoma and prostate carcinoma recruit elevated levels of infiltrating MMP-9positive neutrophils and concomitantly exhibit enhanced levels of angiogenesis and intravasation. Specific inhibition of neutrophil influx by interleukin 8 (IL-8) neutralization resulted in the coordinated diminishment of tumor angiogenesis and intravasation, both of which were rescued by purified neutrophil proMMP-9. However, if neutrophil proMMP-9, naturally devoid of tissue inhibitor of metalloproteinases (TIMP), was delivered in complex with TIMP-1 or in a mixture with TIMP-2, the protease failed to rescue the inhibitory effects of anti-IL8 therapy, indicating that the TIMP-free status of proMMP-9 is critical for facilitating tumor angiogenesis and intravasation. Our findings directly link tumor-associated neutrophils and their TIMP-free proMMP-9 with the ability of aggressive tumor cells to induce the formation of new blood vessels that serve as conduits for tumor cell dissemination. Thus, treatment of cancers associated with neutrophil infiltration may benefit from specific targeting of neutrophil MMP-9 at early stages to prevent ensuing tumor angiogenesis and tumor metastasis. (Am J Pathol 2011, 179:1455-1470; DOI: 10.1016/j.ajpath.2011.05.031)

Cancer progression is accompanied by recruitment of bone marrow-derived cells to the primary tumor, metastatic sites, and pre-metastatic niches. ${ }^{1-5}$ Tumor-recruited lymphoid and myeloid cells, including monocytes/macrophages and neutrophils, promote tumor progression through remodeling of the extracellular matrix (ECM), enhancing tumor cell migration and invasion, and modulating angiogenesis. ${ }^{6-10}$ Among these physiologic processes, tumor angiogenesis is considered critical not only for providing nutrients to developing tumors but for tumor cell dissemination via the hematogenous route.

A specific mechanism by which infiltrating myeloid cells contribute to tumor angiogenesis involves a matrix metalloproteinase-9 (MMP-9)-mediated angiogenic switch. ${ }^{11,12}$ Tumor-associated monocytes/macrophages, mast cells, and neutrophils are all MMP-9-producing leukocytes, which to varying degrees have been linked to tumor-induced angiogenesis. ${ }^{13-22}$ The persistent presence of macrophages in primary tumors throughout cancer progression has led to the notion that non-tumor cell-derived MMP-9 that functionally contributes to angiogenesis and/or tumor dissemination is produced by this leukocyte type. ${ }^{7,14,23-25}$ In contrast, there is an apparent hindrance in demonstrating that neutrophils and neutrophil MMP-9

Supported by the National Institutes of Health $(\mathrm{NIH})$ grants R01 CA 129484 and R01 CA 105412 (J.P.Q.), NIH/National Center for Research Resources/Scripps Translational Science Institute grant UL1 RR025774 (Pilot Award to E.I.D.), a fellowship from Max Kade Foundation (B.S.), and NIH National Cancer Institute training grant 5T32CA077109-10 (E.M.B.).

Accepted for publication May 17, 2011.

E.M.B., B.S., and T.A.K. contributed equally to this work.

Supplemental material for this article can be found on http://ajp. amjpathol.org or at doi: 10.1016/j.ajpath.2011.05.031.

Address reprint requests to Elena I. Deryugina, Ph.D., Department of Cell Biology, The Scripps Research Institute, 10550 N Torrey Pines Rd., La Jolla, CA 92037. E-mail: deryugin@scripps.edu. 
are critical for tumor progression because metastatic spread is usually measured at late stages of tumor development, when the short-lived neutrophils are not readily detectable and macrophages are often the predominant type of tumor-associated leukocytes. ${ }^{26}$ Therefore, relatively few studies have indicated a neutrophil origin for cancer-promoting MMP-9. ${ }^{16,18,20,27}$

The reported MMP-9-triggered angiogenic switch involves proteolytic release from the ECM and subsequent activation of major proangiogenic factors, ie, vascular endothelial growth factor (VEGF) and fibroblast growth factor-2 (FGF-2), which induce proangiogenic signaling responses in the endothelial cells downstream of MMP-9. ${ }^{11,19,28-30}$ Despite overall agreement that MMP-9-induced angiogenesis often correlates positively with tumor dissemination, biochemical mechanisms underlying the MMP-9-mediated aspects of these complex physiologic processes remain unresolved. By using different in vivo models of physiologic tumor-free angiogenesis, we have demonstrated that neutrophil MMP-9 is a potent proangiogenic factor that acts at low nanomolar concentrations as the liberating enzyme of ECM-bound VEGF and FGF-2. ${ }^{29,31}$ Also, neutrophil MMP-9 is released as a proenzyme that must be proteolytically processed and activated before exerting its proangiogenic activity. Whereas all other cells tested, including monocytes and various tumor cells, secrete proMMP-9 in a tight stoichiometric complex with tissue inhibitor of metalloproteinase (TIMP)-1, which negatively regulates its activation, neutrophils are a distinct type of cells that do not express TIMP-1, and, therefore, release their pre-stored proMMP-9 poised for activation. ${ }^{31,32}$ Hence, in contrast to TIMP-complexed MMP-9, neutrophil TIMP-free proMMP-9 can be rapidly activated to exert its catalytic activity, which makes it an exceptionally potent proangiogenic factor acting upstream of FGF-2 and VEGF. 29

In the present study, we investigated whether influx of proMMP-9-delivering neutrophils to primary tumors can coordinately regulate the levels of tumor angiogenesis and tumor cell hematogenous dissemination. By using different in vivo model systems, we established that spontaneous intravasation and metastatic spread of tumor cells selected in vivo for high rates of vascular dissemination depended on their capacity to recruit inflammatory neutrophils, which release angiogenesis-inducing proMMP-9. Conversely, we addressed whether the specific diminishment of neutrophil influx into tumors would result in a corresponding reduction of both tumor angiogenesis and tumor cell intravasation. Finally, we rescued in vivo the inhibitory effects of specific blockage of neutrophil recruitment by exogenous delivery to primary tumors of purified neutrophil proMMP-9. However, no rescue occurred if neutrophil proMMP-9 was stoichiometrically bound to TIMP-1, which dampens activation of the zymogen, ${ }^{29,33,34}$ or used in a mixture with TIMP-2, which inhibits catalysis of the activated enzyme. Together, our findings provide strong evidence that the levels of TIMPfree proMMP-9 delivered by tumor-recruited neutrophils can determine the efficiency of tumor angiogenesis and dissemination.

\section{Materials and Methods}

\section{Human Tumor Cell Lines and Cell Culture}

HT-1080 fibrosarcoma and PC-3 prostate carcinoma parental cell lines were purchased from American Type Culture Collection (Manassas, VA). High- and low-disseminating variants (referred to as hi/diss and lo/diss, respectively) were isolated after serial in vivo passaging of primary tumors (PC-3) or from lung metastases (HT1080) in tumor-bearing chick embryos, and were maintained as previously described. ${ }^{35,36}$

\section{Chick Embryo Assay for Spontaneous Intravasation and Metastasis}

The spontaneous intravasation and metastasis assay in chick embryos was performed as described. ${ }^{35,36}$ Where indicated, the developing tumors were treated topically with 20 to $25 \mu \mathrm{g}$ human IL-8-neutralizing antibody (500P28, PeproTech Inc., Rocky Hill, NJ; or MAB-208, R\&D Systems, Inc., Minneapolis, MN) or normal mouse IgG, applied on days 2 and 4 (HT-1080 variants) or days 2, 4, and 6 (PC-3 variants). When indicated, embryos treated with anti-IL-8 monoclonal antibody (mAb) additionally received either $30 \mathrm{ng}$ per embryo of TIMP-free proMMP-9 purified from isolated human neutrophils, neutrophil proMMP-9 that was complexed with recombinant TIMP-1 and repurified using gelatin Sepharose chromatography to remove excess TIMP-1 (neutrophil proMMP-9-TIMP-1 stoichiometric complex corresponding to $30 \mathrm{ng}$ of proMMP-9), or 30 ng neutrophil MMP-9 with 70 ng recombinant TIMP-2. After the various treatments, primary tumors were excised, weighed, and fixed in $10 \% \mathrm{Zn}$-formalin or embedded in OCT for further analyses. To determine the actual numbers of intravasated cells, portions of the chorioallantoic membrane (CAM) distal to the primary tumor site were harvested on day 5 (HT-1080 variants) or on day 6 or 7 (PC-3 variants), and were analyzed using Alu quantitative PCR. ${ }^{35,36}$

\section{Kidney Capsule Xenotransplantation Model}

All procedures involving animals, such as housing and care, and all experimental protocols were approved by the Institutional Animal Care and Use Committee of The Scripps Research Institute (TSRI). Six- to 8-week-old immunodeficient $n u / n u$ mice were purchased from the TSRI breeding colony. Mice were anesthetized using a mixture of $100 \mathrm{mg} / \mathrm{kg}$ ketamine and $10 \mathrm{mg} / \mathrm{kg}$ xylazine. An incision was made on the left dorsal region to expose the kidney. Firefly luciferase-labeled HT-hi/diss cells ( 0.5 to $1.25 \times 10^{6}$ ) or HT-lo/diss cells $\left(1.0\right.$ to $\left.2.5 \times 10^{6}\right)$ were grafted under the kidney capsule. The kidney was returned to its normal location, and incisions were closed with sutures. At 2 and 10 days after implantation, the mice were injected i.p. with $3 \mathrm{mg}$ luciferin (Caliper Life Sciences, Inc., Hopkinton, MA) and imaged using the IVIS system (Xenogen Corp., Alameda, CA). The mice were sacrificed and dissected at 10 to12 days after tumor cell grafting. Tumor weight was determined by subtracting 
the weight of the contralateral kidney. Lung samples were harvested, fixed in $10 \% \mathrm{Zn}$-formalin for histologic analysis, and frozen for Alu quantitative PCR analysis to determine the number of spontaneously metastasized cells.

\section{Orthotopic Grafting of Prostate Carcinoma Variants}

Eight-week-old male NOD-SCID mice were purchased from the TSRI breeding colony, and surgical procedures were performed as previously described. ${ }^{36}$ A total of $2.5 \times 10^{5} \mathrm{PC}$-hi/diss cells and $5 \times 10^{5} \mathrm{PC}$-lo/diss cells were implanted into the anterior prostate gland. At 4 to 5 weeks after implantation, primary tumors were excised and processed for histologic examination.

\section{CAM Angiogenesis Model}

The collagen onplant model has been previously described in detail. ${ }^{37}$ Tumor cells were incorporated at $1 \times$ $10^{6} / \mathrm{mL}$ neutralized type I rat tail collagen (BD Biosciences, Franklin Lakes, NJ) used at $2.2 \mathrm{mg} / \mathrm{mL}$. When indicated, neutrophil releasate (contents of $10^{6}$ neutrophils $/ \mathrm{mL}$ ), releasate depleted of MMP-9, purified neutrophil proMMP-9 (100 to $300 \mathrm{ng} / \mathrm{mL}$ ), or antibodies neutralizing human IL-8 $(20 \mu \mathrm{g} / \mathrm{mL}$; MAB-208, R\&D Systems, Inc.; 500-P28, PeproTech Inc.) or VEGF (50 $\mu \mathrm{g} / \mathrm{mL}$; AB-293-NA, R\&D Systems, Inc.) were incorporated into collagen mixtures. A subset of onplants containing anti-IL-8 was supplemented with purified neutrophil proMMP-9, proMMP-9-TIMP-1 stoichiometric complex, or proMMP-9 mixed with recombinant TIMP-2. Thirty microliters of collagen mixture was polymerized between two nylon grid meshes to form a collagen "onplant." Five or six collagen onplants were placed on the CAM of 10-day-old embryos developing ex ovo (four to six embryos per variable). When indicated, embryos were treated with $1 \mathrm{mg}$ ibuprofen suspended in $200 \mu \mathrm{L}$ $1 \%$ methyl cellulose and injected into the allantoic cavity at 2 hours before and 2 days after the grafting of onplants containing 1 to $3 \mathrm{ng}$ purified neutrophil TIMP-free proMMP-9. An angiogenic index was determined within 72 to 96 hours for each onplant as the fraction of grids with newly formed blood vessels over the total number of grids scored.

\section{Angiogenesis Assay in Mice}

The assay was performed as previously described. ${ }^{29,36}$ In brief, hi/diss or lo/diss tumor cells were incorporated into 2.0 to $2.5 \mathrm{mg} / \mathrm{mL}$ type I rat tail collagen at a final concentration of $1 \times 10^{6}$ cells $/ \mathrm{mL}$. Approximately 30 to $40 \mu \mathrm{L}$ of the resultant collagen suspensions were polymerized at $37^{\circ} \mathrm{C}$ in $1-\mathrm{cm}$ long silicon tubes, making an "angiotube." A total of four angiotubes were inserted into two air pockets created on both dorsal sides of anesthetized nu/nu mice. After 12 days (HT-1080 variants) or 19 days (PC-3 variants), the mice were sacrificed, and skin flaps with angiotubes were exposed and photographed. After careful excision, the contents of the angiotubes were flushed out and lysed in modified radioimmunoprecipitation assay buffer. The levels of angiogenesis in the angiotubes determined using Western blot analysis for the endothelial marker CD31 correlated well with hemoglobin content determined using the QuantiChrom Hemoglobin Assay Kit (BioAssay Systems LLC, Hayward, CA). Therefore, the hemoglobin content was used as a measurement of angiogenesis, similar to the approach commonly used to quantify the extent of neovascularization in Matrigel plugs (BD Biosciences). ${ }^{38}$

\section{Isolation of Human Neutrophils and Analysis of Purity of Neutrophil Fraction}

Human peripheral blood was collected from healthy donors in accordance with the protocol approved by the Institutional Review Board of TSRI. To isolate human neutrophils, heparinized blood from healthy donors was diluted $1: 3$ with $0.9 \% \mathrm{NaCl}$ and centrifuged at $200 \times g$ to wash out platelets. Mononuclear cells were separated from granulocytes using a Histopaque Ficoll 1.077 gradient by centrifugation at $400 \times g$ for 45 minutes at ambient temperature. The bottom fraction containing granulocytes and erythrocytes was diluted with $0.9 \%$ $\mathrm{NaCl}$ and mixed with $6 \%$ dextran, and erythrocytes were allowed to sediment to the bottom of the tube. The remaining red blood cells in the supernatant were either lysed with buffer containing $0.15 \mathrm{mmol} / \mathrm{L} \mathrm{NH}{ }_{4} \mathrm{Cl}, 12$ $\mathrm{mmol} / \mathrm{L} \mathrm{NaHCO}_{3}$, or $100 \mathrm{mmol} / \mathrm{L} \mathrm{Na} \mathrm{NDTT}_{2}$ (all from Sigma-Aldrich Corp., St. Louis, MO) or with $0.2 \% \mathrm{NaCl}$ for 30 seconds, followed by addition of an equal volume of $1.6 \%$ $\mathrm{NaCl}$ to restore isotonicity. After the last centrifugation, the pellet containing the neutrophils was resuspended in PBS at $1 \times 10^{7} \mathrm{cells} / \mathrm{mL}$.

The $97 \%$ to $98 \%$ purity of the neutrophil fraction was confirmed via differential staining with Wright-Giemsa using the Hema-3 Kit (Fisher Scientific, Pittsburgh, PA). The percentage of neutrophils, eosinophils, monocytes, and lymphocytes was determined after several independent neutrophil fractionations. Cytomorphologic analysis of eight independent fractionations indicated that approximately $97 \%$ to $98 \%$ of isolated leukocytes were neutrophils, whereas less than $0.5 \%, 1.8 \%$, and $0.3 \%$ constituted monocytes, eosinophils, and lymphocytes, respectively. Isolated neutrophils were also analyzed for expression of granulocytic antigen CD66c using flow cytometry. Isolated neutrophils were incubated with 5 $\mu \mathrm{g} / \mathrm{mL}$ anti-human $\mathrm{CD66c}$ murine mAb (clone B6.2/ CD66; BD Biosciences) or normal mouse IgG (Jackson ImmunoResearch Laboratories, Inc., West Grove, PA), incubated with fluorescein isothiocyanate-conjugated secondary antibody and analyzed using a flow cytometer (Becton Dickinson \& Co., Franklin Lakes, NJ). Smears from human peripheral blood or isolated neutrophils were immunostained using rabbit polyclonal antibody (Abcam, Inc., Cambridge, MA) and murine mAb 8-3H specific for human MMP-9 (generated in our laboratory). 


\section{Purification of Neutrophil ProMMP-9}

Release of neutrophil granules, gelatin Sepharose purification of neutrophil proMMP-9, its complex formation with human recombinant TIMP-1, and repurification of TIMP-1-proMMP-9 complexes were performed as previously described. ${ }^{29,31}$

\section{Neutrophil Chemotaxis Assay}

Neutrophil migration in vitro was analyzed using Transwell inserts with 3- $\mu \mathrm{m}$ membrane pore size (Corning Life Sciences, Corning, Inc., Corning, NY). A total of $2 \times 10^{5}$ human neutrophils were placed in $100 \mu \mathrm{L}$ serum-free Dulbecco's modified Eagle's medium per insert and allowed to migrate for 2 to 4 hours to the bottom chamber filled with $600 \mu \mathrm{L}$ tumor cell-conditioned medium containing 10 to $20 \mu \mathrm{g} / \mathrm{mL}$ control mouse IgG or anti-human IL-8 neutralizing antibody (MAB-208; R\&D Systems, Inc.). The number of migrated neutrophils was determined either by counting the cells at the bottom chamber with a hemocytometer or by quantifying the cells located at the bottom of the well in six randomly imaged fields.

\section{Isolation of Chicken Neutrophils from Peripheral Blood and Immunostaining for Chicken MMP-9}

Chicken neutrophils (heterophils) from EDTA-buffered peripheral blood of adult roosters or 15- to 17-day-old chick embryos were isolated from the 1.119 phase of a two-phase (1.077 and 1.119) Histopaque gradient (Sigma-Aldrich Corp.). To analyze MMP-9 expression, the isolated neutrophils were fixed with ice cold methanol and stained with $4 \mu \mathrm{g} / \mathrm{mL}$ mouse mAb K2-F10 or C2-E10 specific for chicken MMP-9 (both produced in our laboratory), followed by incubation with the secondary fluorescein isothiocyanate-conjugated anti-mouse antibody.

\section{Immunohistochemistry and Blood Vessel Staining}

Primary CAM tumors and murine xenografts were excised and frozen in OCT or fixed in 10\% Zn-formalin, and paraffin-embedded. Deparaffinized tissue sections or cryosections fixed in cold methanol were treated with $0.3 \%$ hydrogen peroxide and blocked with PBS supplemented with $1 \%$ bovine serum albumin and $1 \%$ normal goat serum. Human tumor cells were stained with 1 $\mu \mathrm{g} / \mathrm{mL}$ anti-human CD44 mAb 29-7 (generated in our laboratory). Chicken neutrophils were stained in frozen sections of CAM tumors with $2 \mu \mathrm{g} / \mathrm{mL}$ rabbit anti-chicken MMP-9 or murine mAb K2-F10. Monocytes were highlighted with rabbit anti MMP-13 polyclonal antibody (Abcam, Inc.). In paraffin sections from orthotopic PC-3 xenografts, murine neutrophils were stained with 4 $\mu \mathrm{g} / \mathrm{mL}$ rat anti-mouse Ly6G mAbs Gr-1 (eBioscience, Inc., San Diego, CA) or 1A8 (BD Biosciences), monocytes/macrophages were highlighted with $4 \mu \mathrm{g} / \mathrm{mL}$ Mac-2 mAb (M3/38; Cederlane Laboratories, Ltd., Burlington, ON, Canada), and blood vessels were visual- ized by staining endothelial cells with $5 \mu \mathrm{g} / \mathrm{mL}$ rabbit polyclonal anti-CD31 antibody (Abcam, Inc.). Bound primary antibodies were detected using corresponding biotinylated secondary antibodies (Vector Laboratories, Inc., Burlingame, CA).

The chicken vasculature was highlighted with biotinylated Sambucus nigra agglutinin (SNA) and visualized using avidin-D horseradish peroxidase conjugate (Vector Laboratories, Inc.). Counterstaining was performed using Mayer's hematoxylin. In live embryos, the CAM vasculature was highlighted by rhodamine-conjugated Lens culinaris agglutinin (LCA) (Vectors Labs, Burlingame, CA). Images were captured using an Olympus BX60 microscope equipped with a digital DVC video camera (Olympus Corp., Tokyo, Japan) and processed using Adobe Photoshop 6.0 software (Adobe Systems, Inc., San Jose, CA).

To quantify the density of neutrophils and monocytes and levels of neovascularization, serial images were obtained at original magnification $\times 200$, and the number of MMP-9positive neutrophils, MMP-13-positive monocytes, or lumen-containing vessels was determined per tumor-filled area in 10 to 20 individual images per tumor, with a total of 3 to 5 individual tumors analyzed per variable.

To analyze pericyte coverage of tumor vasculature, deparaffinized sections of CAM primary tumors were double-stained with anti-CD31 antibody to highlight the endothelial cells and with anti-desmin mAb DE-U-10 (Abcam, Inc.) to highlight the pericytes. After incubation with corresponding species-specific fluorescent-tagged secondary antibodies, bound antibodies were visualized using fluorescence microscopy. Cell nuclei were stained with $1 \mu \mathrm{g} / \mathrm{mL}$ DAPI (Molecular Probes, Inc., Eugene, OR).

Double immunostaining of PC-hi/diss xenografts for MMP-9 and Ly6G antigens was performed in frozen sections incubated with $2 \mu \mathrm{g} / \mathrm{mL}$ rabbit MMP-9 antibody and $4 \mu \mathrm{g} / \mathrm{mL}$ rat $\mathrm{mAb} \mathrm{Gr}-1$ or $1 \mathrm{~A} 8$, followed by incubation with the secondary Texas Red-conjugated anti-rabbit and Alexa Fluor 488-conjugated anti-rat antibodies (Jackson ImmunoResearch Labs, Inc). Cell nuclei were highlighted using DAPI.

\section{Analysis of Apoptosis and Necrosis in Primary Tumors}

Primary CAM tumors were stained according to the manufacturer's instructions using the ApopTag Peroxidase In Situ Apoptosis Detection Kit (Chemicon International, Inc., Temecula, CA), which enabled clear differentiation of cells undergoing apoptosis from cells undergoing necrosis. In tumor sections, the positively stained tumor cells become brown after addition of a chromogenic peroxidase diaminobenzidine substrate. The positively stained cells were defined as apoptotic if they exhibited typical apoptotic features, eg, they were smaller than the nonstained cells, and contained a condensed nucleus and a thin rim of cytoplasm. In contrast, large positively stained cells with swollen cytoplasm and marginal nuclear condensation were defined as necrotic tumor cells. 


\section{Cytokine Antibody Array}

Serum-free conditioned medium was collected from PChi/diss or HT-hi/diss cell monolayers after 48 hours of incubation. Loading was normalized to cell number per culture at collection of conditioned medium. The RayBio Human Cytokine Antibody Array III (RayBiotech, Inc., Norcross, GA) was performed according to the manufacturer's instructions. Densitometry of the positive spots was quantified using an Alpha Imager (Alpha Innotech Corp., San Leandro, CA).

\section{Data Analysis and Statistics}

Data processing and statistical analyses were performed using GraphPad Prism Software (GraphPad Software, Inc., San Diego, CA). Data are given as mean \pm SEM (bar graphs) or median (scattergrams) from a representative experiment or several normalized experiments, in which percent changes were calculated from the pooled fold differences determined by taking ratios of numerical values for individual embryos or mice over the mean of the control group. The Student's $t$-test or Mann-Whitney $U$ test was used to determine significance $(P<0.05)$ of differences between data sets. Numbers of animals and tissue samples analyzed and the number of experiments performed is indicated in the figure legends.

\section{Results}

\section{Tumor Cell Intravasation and Metastasis in Avian and Mammalian Models}

Human HT-1080 fibrosarcoma and PC-3 prostate carcinoma variants with distinct disseminating abilities (ie, lo/ diss and hi/diss tumor cells) were previously isolated via in vivo selection in chick embryos. ${ }^{35,36}$ The quantitative comparative analysis of tumor growth and intravasation in the avian spontaneous metastasis model indicates substantial differentials (50- to 100-fold) in the intravasation capacity between hi/diss and lo/diss cells from both tumor cell types despite the ability to form primary tumors of similar size (Figure 1, A and B).

The clear differential in intravasation and metastasis exhibited by hi/diss and lo/diss cells can be recapitulated in mammalian models. Thus, orthotopic xenotransplantation of PC-hi/diss and PC-lo/diss cells into the anterior prostate gland of immunodeficient mice confirmed the differential dissemination behavior of this congenic pair. ${ }^{36}$ The selected fibrosarcoma variants also exhibit corresponding dissemination differences in a mammalian model, in which primary tumors develop under the kidney capsule of immunodeficient mice. Within 10 to 12 days after surgical implantation, both HT-hi/diss and HT-lo/diss formed primary tumors of comparable size but demonstrated a significant difference between the number of human cells that had metastasized to the lungs (Figure 1, C-E). Therefore, tumor cell variants originally selected in the chick embryo model accurately recapitulate their dif- ferential capacities for spontaneous metastasis in mammalian models.

\section{Ability of Tumor Cells to Intravasate and Metastasize Correlates with Their Angiogenic Potential}

That the selection of tumor variants for high levels of intravasation and metastasis resulted in the isolation of cells with high angiogenic potential was first verified using the CAM angiogenesis assay. Comparative analysis of angiogenic potentials exhibited by hi/diss versus lo/ diss variants of both fibrosarcoma and prostate carcinoma indicated that the propensity of tumor cells to intravasate and disseminate correlated well with their angiogenic potential (Figure 2A). The difference in angiogenic potential exhibited by hi/diss tumor variants in the avian model were also recapitulated in the mouse angiotube model (Figure 2B). Together, these results are consistent with the notion that the ability of tumor cells to spontaneously intravasate and disseminate is positively linked to their angiogenesis-inducing capacity.

We next determined whether the angiogenic potentials of hi/diss and lo/diss variants of HT-1080 fibrosarcoma and PC-3 prostate carcinoma were reflected in corresponding high and low levels of angiogenesis in primary CAM tumors. Staining of blood vessels with SNA in HT tumors (Figure 2C) or rhodamine-tagged LCA in PC tumors (Figure 2D) indicated that, despite similar size of primary tumors, angiogenesis was more advanced in hi/diss tumors than in their lo/diss counterparts. Because fluorescent LCA was administered into live embryos, followed by microscopy of non-fixed CAM tumors, the high levels of fluorescence and, therefore, vessel perfusion observed in PC-hi/diss tumors (Figure 2D) indicated that enhanced angiogenesis was accompanied by development of intratumoral vasculature with functional microcirculation. Quantitative analysis of the density of lumencontaining blood vessels in primary CAM tumors stained with SNA confirmed that HT-hi/diss cells and PC-hi/diss cells were fourfold to fivefold more potent in induction of angiogenesis than their corresponding lo/diss counterparts (scattergrams in Figure 2, C and D, respectively) $(P<0.001)$. It is worth noting that lower levels of angiogenesis in primary lo/diss tumors are not associated with higher levels of apoptosis or necrosis (see Supplemental Figure S1 at $h$ ttp://ajp.amjpathol.org), most probably because of the efficient gas exchange and nutrient supply provided for developing tumors by the CAM mesoderm vasculature and ectoderm capillary plexus.

\section{Recruitment of MMP-9-Positive Neutrophils to Highly Disseminating Primary Tumors}

We next sought to establish whether the angiogenesis observed in primary tumors developing from hi/diss tumor variants was concomitant with the influx of inflammatory leukocytes delivering MMP-9, which we had previously demonstrated to be potently angiogenic in nontumor model systems. ${ }^{29,31}$ Immunohistochemical 
A

HT-1080 Variants

Primary Tumor
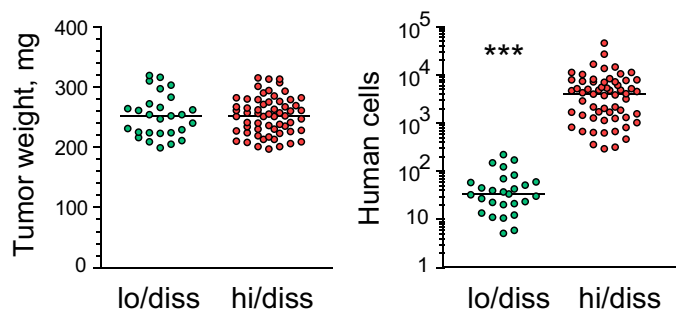

C

Tumor Growth

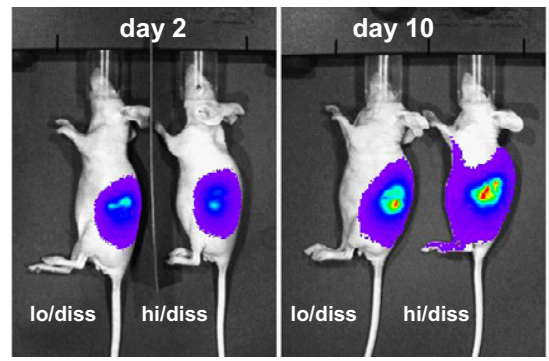

day 12

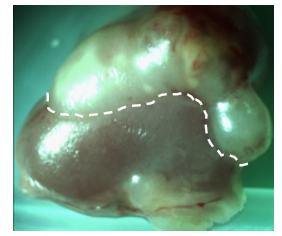

HT-lo/diss

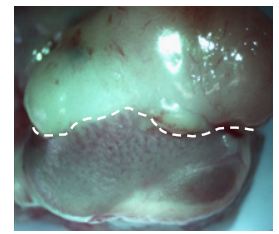

HT-hi/diss
D
B

PC-3 Variants

Primary Tumor Intravasation
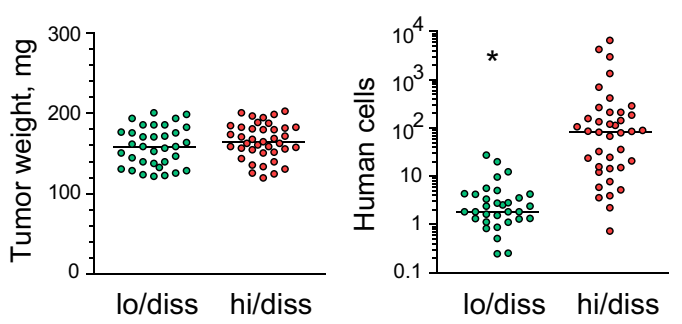

$\mathrm{E}$

\section{Kidney}
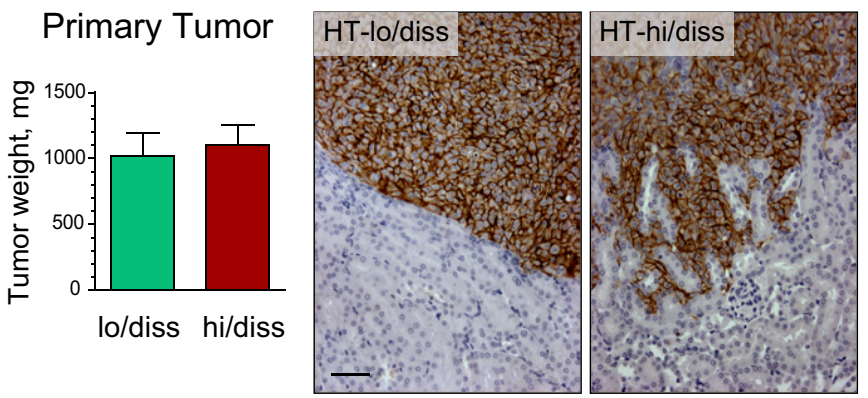

Lung metastasis

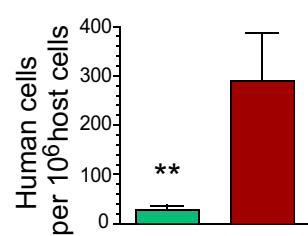

lo/diss hi/diss
Lung

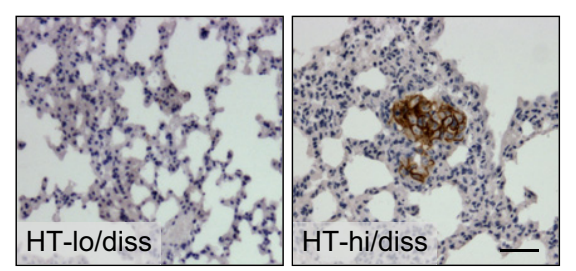

Figure 1. Dissemination capacity of HT-1080 and PC-3 variants in spontaneous metastasis models. A and B: Tumor growth and intravasation in the chick embryo model. The levels of intravasation of HT-1080 fibrosarcoma (A) or PC-3 prostate carcinoma (B) variants were determined using Alu quantitative PCR in the embryos bearing primary tumors of similar size. Note the logarithmic scale in the intravasation scattergrams. The scattergrams represent cumulative data from nine independent experiments involving grafting of HT-lo/diss $(n=27)$ and HT-hi/diss $(n=60)$ and five independent experiments involving grafting of PC-lo/diss $(n=34)$ and PC-hi/diss $(n=39)$. ${ }^{* * * *} P<0.001$, two-tailed Student's $t$-test. C-E: Tumor growth and metastasis of HT-1080 variants in the kidney capsule xenograft model. C: Tumor development of HT-lo/diss and HT-hi/diss variants was analyzed by noninvasive imaging of firefly luciferase-tagged cells using the IVIS system at 2 and 10 days after cell implantation under the renal capsule of immunodeficient mice (top panels). Kidneys with developed tumors were excised from the mice on day 12. Dotted line demarcates the border between the primary tumor and the top of the kidney (bottom panels). D: Efficiency of the primary tumor growth was determined after subtracting the weight of the contralateral kidney. Data from one of six individual experiments involving mice bearing HT-lo/diss $(n=4)$ and HT-hi/diss $(n=6)$ tumors (top graph). Levels of lung metastasis in mice bearing HT-lo/diss and HT-hi/diss tumors were quantified using Alu quantitative PCR (bottom graph). Data are given as mean \pm SEM from a representative experiment. ${ }^{* *} P<0.05$, two-tailed Mann-Whitney $U$-test. E: Immunohistologic detection of human tumor cells within mouse tissues was performed using human-specific CD44 antibody (brown). Top panels: High levels of tumor cell invasion in HT-hi/diss xenografts as compared with their lo/diss counterparts. Scale bar $=50 \mu \mathrm{m}$. Bottom panels: Metastatic cells (brown) were detected in the lung from a mouse bearing an HT-hi/diss tumor. Scale bar $=50 \mu \mathrm{m}$.

staining of primary tumors clearly demonstrated that both HT-hi/diss and PC-hi/diss variants exhibited enhanced levels of MMP-9-positive leukocytes, in particular at the tumor border (Figure 3, A and B). Morphologic evaluation indicated that essentially all MMP-9-positive cells in CAM tumors contained multilobulated nuclei characteristic of mature granulocytes. In the CAM mesoderm, cells with monocyte/macrophage characteristics, ie, bean-shaped nuclei and abundant cytoplasm, seem to be MMP-9-negative or express MMP-9 barely above background, as opposed to neutrophils that are highly positive for MMP-9 (see Supplemental Figure S2A at http://ajp.amjpathol.org). Immunocytochemical staining of neutrophils isolated from the peripheral blood of chick embryos or adult roosters confirmed high levels of MMP-9 within their cytoplasmic granules (see Supplemental Figure S2, B and C at http://ajp.amjpathol.org).

Detailed quantitative kinetic analysis of CAM tumors indicated an almost exponential increase in the influx of MMP-9-positive neutrophils into HT-hi/diss tumors, in contrast to their HT-lo/diss counterparts, resulting in a more than fourfold neutrophil differential by day 5 (Figure 3A, bar graph). A similar differential in the influx of MMP-9-positive neutrophils was observed in the $\mathrm{PC}-3$ prostate carcinoma dissemination variants at 5 or 6 days after grafting (Figure 3B, bar graph), affirming the neutrophil influx link with an epithelial cancer. 
A

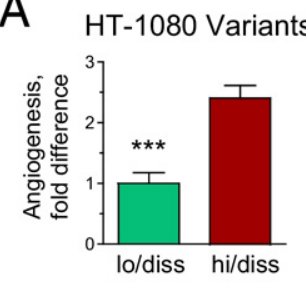

PC-3 Variants

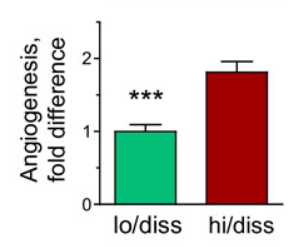

C

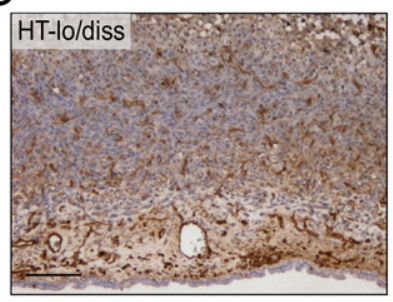

D

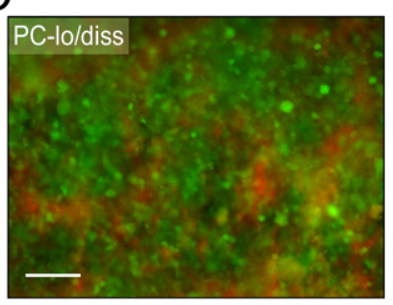

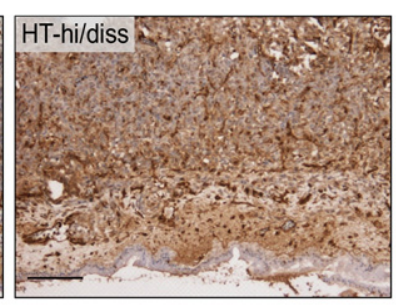

B HT-lo/diss

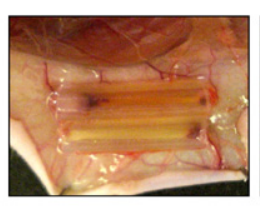

PC-lo/diss

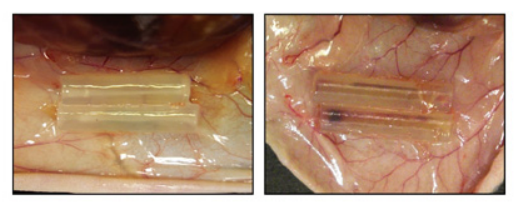

HT-hi/diss

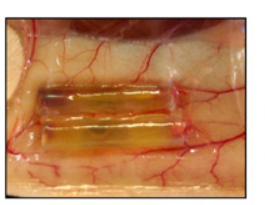

PC-hi/diss

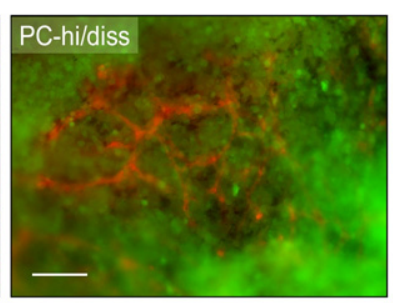

Tumor Angiogenesis

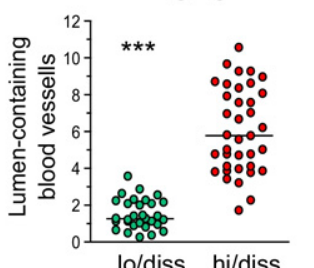

Tumor Angiogenesis

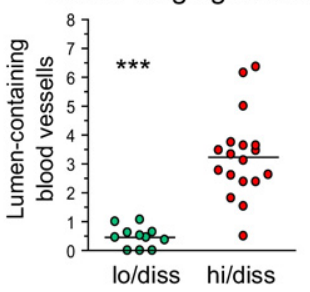

Figure 2. Angiogenesis induced by HT-1080 and PC-3 dissemination variants in avian and mammalian models. A: Angiogenic potential in the chick embryo collagen onplant model. Angiogenic potential of hi/diss variants of HT-1080 fibrosarcoma and PC-3 prostate carcinoma were quantified as fold differences between angiogenic indexes determined in individual onplants compared with their corresponding lo/diss counterparts. Cumulative data from three HT1080 and five PC-3 independent experiments involving 30 to 79 individual onplants per variant are given as mean \pm SEM. ${ }^{*} P<0.05$, ${ }^{* * * *} P<$ 0.001 , two-tailed Student's $t$-test. B: Angiogenic potential in the mouse angiotube model. Panels depict blood vessels converging onto angiotubes. Bar graphs on the right depict fold difference in angiogenesis levels between tumor variants as determined by hemoglobin content in the tubes from three HT-1080 and two PC-3 independent experiments involving 7 to 18 mice per tumor variant. C and $\mathbf{D}$ : Tumor angiogenesis in primary CAM tumors. Primary tumors that developed in the chick embryos from HT-1080 variants $(\mathbf{C})$ or PC-3 variants $(\mathbf{D})$ were stained with endothelial cell-specific lectins (SNA or LCA) to visualize blood vessels. Immunohistochemical staining of HT-lo/diss and HT-hi/diss tumors with SNA lectin (C) was followed by quantifying the density of lumen-containing vessels, presented as the scattergram on the right. Scale bar $=100 \mu \mathrm{m}$. Number of sections analyzed from three to five individual tumors: HTlo/diss, $n=30$; HT-hi/diss, $n=38$. Tumor blood vessels in primary PC-lo/diss and PC-hi/ diss tumors were highlighted with red fluorescent-tagged LCA injected into live embryos (D). Scale bar $=100 \mu \mathrm{m}$. Quantification of tumor angiogenesis was performed in SNA-stained tumor sections. Number of sections was analyzed from four or five individual tumors: PC-lo/diss, $n$ $=11 ;$ PC-hi $/$ diss, $n=18 .{ }^{\text {***** }} P<0.001$, twotailed Student's $t$-test.
The enhanced influx of neutrophils into primary tumors concomitant with enhanced angiogenic and metastatic potential was also demonstrated in a mammalian model for the prostate carcinoma dissemination variants. Four to 6 weeks after orthotopic implantation into the prostate tissue of SCID mice, PC-lo/diss and PC-hi/diss xenografts yielded a substantial differential in both the influx of inflammatory neutrophils and tumor angiogenesis, as judged by staining for Gr-1/Ly6G $\mathrm{G}^{\text {high }}$ and CD31-positive cells, respectively (Figure $3 \mathrm{C}$ ). Double-immunofluorescent staining indicated that within PC-hi/diss prostate tumors, $93.7 \% \pm 1.3 \%(n=211)$ of $\mathrm{Gr}-1 / \mathrm{Ly} 6 \mathrm{G}^{\text {high }}$ cells were positive for MMP-9, and essentially all MMP-9-positive cells contained multilobulated nuclei, consistent with their granulocytic lineage of differentiation (Figure 3D). Furthermore, complementary immunostaining with $\mathrm{mAb}$ $1 \mathrm{~A} 8$, strictly specific for Ly6G, confirmed that $91.2 \% \pm$ $2.7 \%$ of $1 \mathrm{~A} 8 /$ Ly6G-positive granulocytes $(n=377)$ in PC-hi/diss xenografts were also intensely positive for murine MMP-9 (see Supplemental Figure S3 at http://ajp. amjpathol.org).

Together with the findings in the CAM model, these results from the mouse tumor model are consistent with the notion that the high propensity of certain tumor cells to actively disseminate correlates strongly with their pronounced ability to induce angiogenesis via attraction of inflammatory neutrophils, which are equipped for immediate delivery of presynthesized angiogenic MMP-9 into the microenvironment of the developing tumor.

\section{Functional Role of Neutrophil proMMP-9 in Tumor-Induced Angiogenesis}

Because the angiogenic capacity of the tumor variants positively correlated with their ability to recruit neutrophils bearing highly angiogenic proMMP-9, it was hypothesized that supplementation of neutrophil-derived MMP-9 to lo/diss cells might increase their angiogenic potential. Therefore, HT-lo/diss and PC-lo/diss cells were incorporated into three-dimensional (3D) collagen gels with or without proMMP-9 purified from human neutrophils to measure levels of induced angiogenesis. Indeed, the low angiogenic potentials exhibited by the lo/diss tumor variants were increased to the levels of their corresponding hi/diss counterparts by addition of purified neutrophil 
A

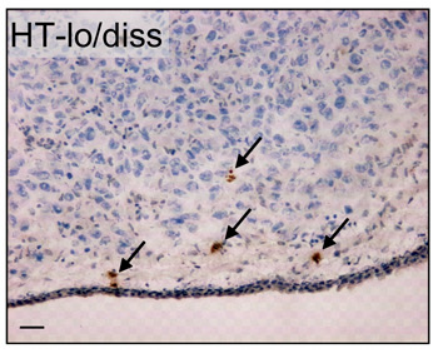

HT-1080 Variants

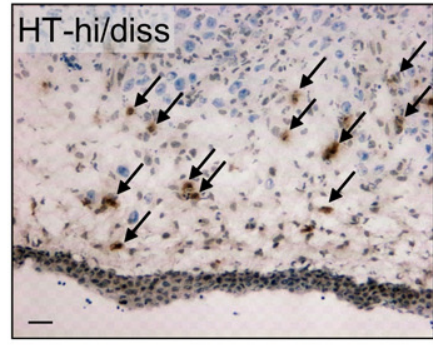

HT-1080 Variants

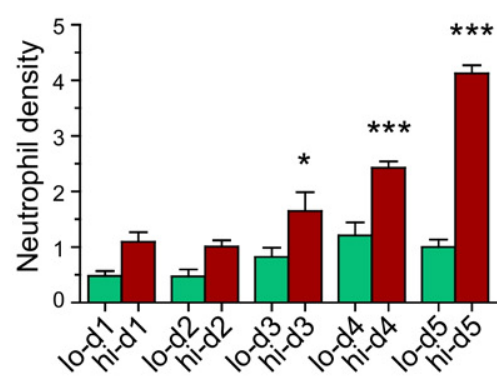

B

PC-3 Variants
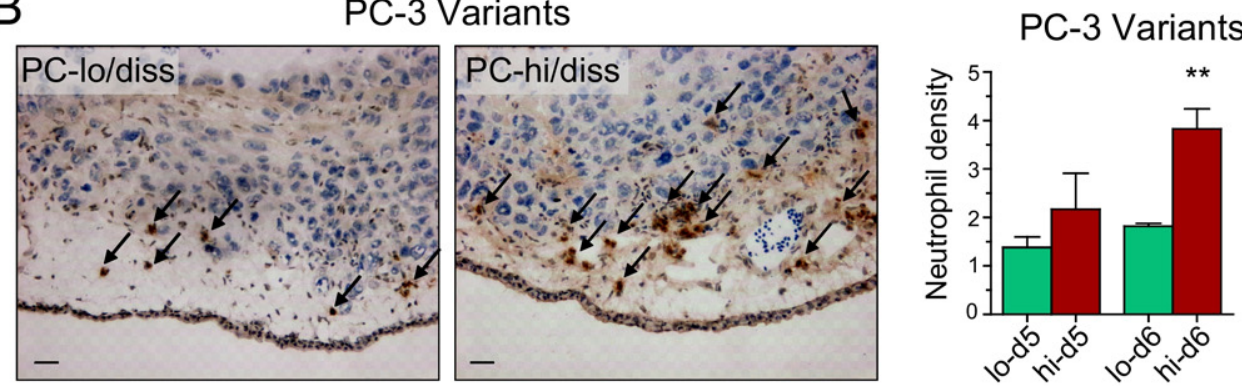

C

H\&E

CD44

Gr-1(Ly6G high $)$

CD31
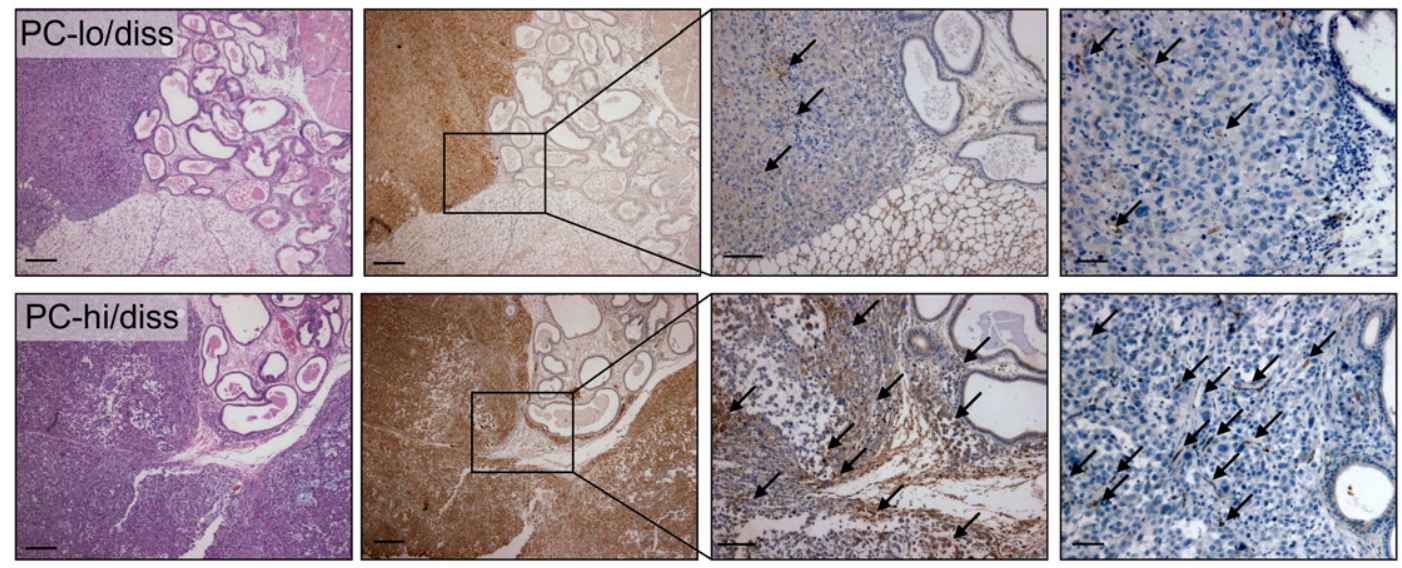

\section{Gr-1(Ly6Ghigh)}

MMP-9

Gr-1 (Ly6Gigh)/MMP-9/DAPI
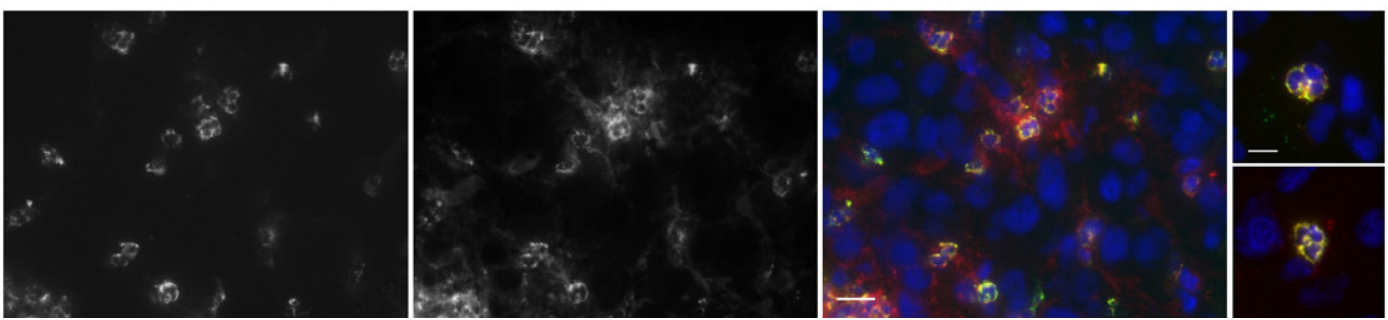

Figure 3. Levels of neutrophil influx into primary tumors correlate with tumor cell dissemination potential. A and B: Tumor-recruited neutrophils immunostained with MMP-9-specific antibody (brown) in CAM primary tumors developed from HT-1080 variants (A) and PC-3 variants (B). Arrows point to some of the stained neutrophils. Scale bars $=25 \mu \mathrm{m}$. Bar graphs: Density of MMP-9-positive neutrophils in CAM tumors harvested at different times after grafting of HT-1080 and PC-3 dissemination variants. Three to eight individual tumors were analyzed at each time point. ${ }^{*} P<0.05$, ${ }^{* *} P<0.01$, and ${ }^{* * * *} P<0.001$, two-tailed Student's $t$-test. C: Levels of neutrophil infiltration and angiogenesis in prostate carcinoma orthotopic xenografts correlate with dissemination capacity of lo/diss (upper panels) and hi/diss (bottom panels) tumor variants. Tissue sections from tumors harvested at 4 to 6 weeks after implantation were stained with H\&E, human-specific CD44 antibody to discriminate human tumor cells, Gr-1 antibody to detect Ly6G-positive murine neutrophils, and CD31 antibody to highlight blood vessels. Arrows point to some of the Gr-1-positive neutrophils and CD31-positive blood vessels. Scale bars: $200 \mu \mathrm{m}$ for H\&E and CD 44 staining; $100 \mu \mathrm{m}$ for Gr-1 staining; $50 \mu \mathrm{m}$ for CD31 staining. D: Immunohistologic staining of PC-hi/diss xenografts for Ly6G ${ }^{\text {high }}$ with Gr- 1 rat mAb and for MMP-9 with rabbit polyclonal antibody. The merged image, also depicting cell nuclei stained with DAPI, indicates that all Gr-1/Ly6G $\mathrm{G}^{\text {high }}$-positive cells are MMP-9-positive neutrophils. Scale bar $=25 \mu \mathrm{m}$. Two merged images on the right depict individual tumor-associated neutrophils at higher magnification of $\times 630$ to illustrate characteristic multilobulated nuclei. Scale bar $=10 \mu \mathrm{m}$. 
A

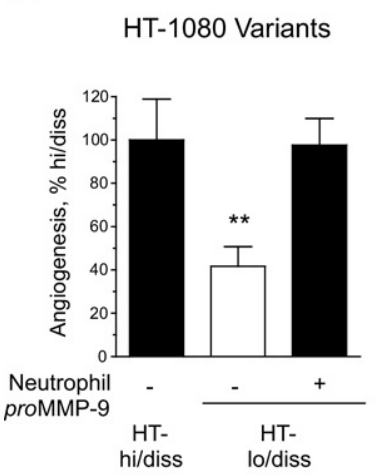

B

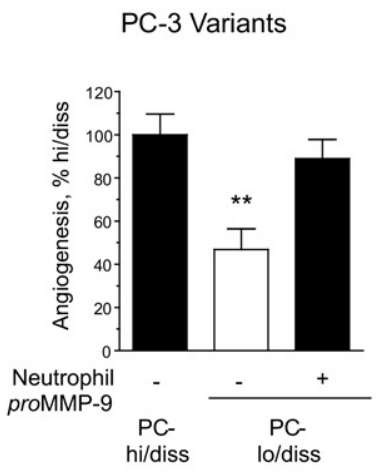

C

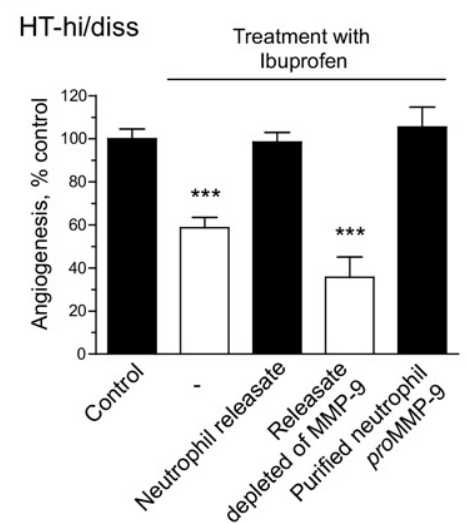

$\mathrm{D}$

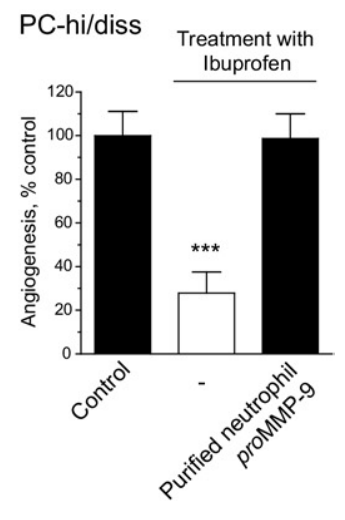

Figure 4. Angiogenic potential of HT-1080 and PC-3 dissemination variants is regulated by neutrophil proMMP-9. A and B: Angiogenesis levels induced by hi/diss and lo/diss variants of HT-1080 fibrosarcoma (A) and PC-3 prostate carcinoma (B) were determined in the CAM collagen onplant model in three to six independent experiments. Low levels of angiogenesis manifested by both types of lo/diss cells were restored by exogenous addition of 2 ng purified neutrophil promMP-9. Forty-six to 80 individual onplants were analyzed in each group containing fibrosarcoma cells (A), and 20 to 23 individual onplants were analyzed in each group containing prostate carcinoma cells (B). Data are expressed as a percentage of angiogenesis determined in a corresponding hi/diss group and represent mean \pm SEM. ${ }^{* *} P<0.005$, two-tailed Student's $t$-test. $\mathbf{C}$ and $\mathbf{D}$ : Levels of angiogenesis diminished by the anti-inflammatory drug ibuprofen were rescued in HT-hi/diss (C) and PC-hi/diss (D) variants by exogenously added neutrophil releasate or 2 ng purified neutrophil promMP-9 but not by neutrophil releasate specifically depleted of MMP-9 gelatinase by affinity chromatography. Twenty to 94 individual onplants were analyzed for each group containing fibrosarcoma cells (C), and 16 to 22 individual onplants were analyzed in each group containing prostate carcinoma cells (D). Data are expressed as a percentage of angiogenesis determined in the control group (no treatment with ibuprofen and no additives) and represent mean \pm SEM. ${ }^{* * * *} P<0.001$, two-tailed Student's $t$-test.

proMMP-9 (Figure 4, A and B), strengthening the notion that neutrophils and their released proMMP-9 can directly affect tumor angiogenesis.

To further confirm that neutrophil proMMP-9 has a direct functional role in tumor-induced angiogenesis, overall inflammatory responses were inhibited with ibuprofen, a potent anti-inflammatory drug that efficiently blocks leukocyte infiltration in chick embryo models. ${ }^{39}$ Ibuprofen significantly inhibited tumor-induced angiogenesis in both $\mathrm{HT}$-hi/diss onplants by $40 \%$ and PC-hi/diss onplants by $70 \%$ (Figure 4, C and D). However, if HT-hi/disscontaining onplants were additionally supplemented with the secretory contents released by human neutrophils (herein referred to as releasate), ibuprofen-inhibited angiogenesis was fully restored. When this neutrophil releasate was specifically depleted of proMMP-9 by affinity chromatography, its rescuing ability was completely abolished, demonstrating that neutrophil proMMP-9 was the critical proangiogenic factor supplied by degranulated neutrophils. In agreement with this, proMMP-9 purified from the neutrophil releasate and supplemented at low to subnanomolar levels $(0.5$ to $1.0 \mathrm{nmol} / \mathrm{L})$ into both HT-hi/diss and PC-hi/diss onplants completely restored ibuprofen-inhibited angiogenesis (Figure 4, C and D). Together, these findings point to neutrophil proMMP-9 as a potent angiogenic factor that can be rapidly released by neutrophils that infiltrate developing tumors.

\section{Neutrophil Recruitment Is Required for High Levels of Tumor Angiogenesis and Intravasation}

To demonstrate that influxing MMP-9-positive neutrophils have a functional and concomitant role in tumor cell angiogenesis and intravasation, we took advantage of specific inhibition of neutrophil infiltration by blocking one of their major chemoattractants, IL-8 (CXCL8). ${ }^{40,41}$ Cyto- kine arrays confirmed that both $\mathrm{HT}$ and $\mathrm{PC}$ dissemination variants produce neutrophil-attracting IL-8 (see Supplemental Figure S4 at http://ajp.amjpathol.org); thus, IL-8 neutralization should enable highly specific inhibition of neutrophil influx. Previously published data demonstrating that avian neutrophils express IL-8 receptor CXCR $1^{42}$ and respond to human IL-8 have provided a strong rationale for a series of IL-8-neutralizing experiments with the objective of mechanistically linking the levels of neutrophil influx into hi/diss primary tumors with the levels of tumor angiogenesis and intravasation.

IL-8-neutralizing antibody efficiently inhibited neutrophil migration induced by PC-hi/diss-conditioned medium in vitro. In primary tumors in vivo, functional depletion of IL-8 prevented the influx of host neutrophils but did not significantly affect recruitment of monocytes/macrophages. Furthermore, anti-IL-8 therapy inhibited tumorinduced angiogenesis in collagen onplants and was as effective as anti-VEGF therapy (see Supplemental Figure S5 at http://ajp.amjpathol.org).

In the developing tumors, anti-IL-8 antibody demonstrated no significant effects on overall primary tumor development, as judged by staining with human-specific CD44 antibody and tumor weight measurements (Figure 5 and Figure 6). However, functional depletion of IL-8 resulted in concomitant inhibition of neutrophil recruitment and tumor angiogenesis (Figures 5A and 6A), quantitatively presented in the corresponding bar graphs (Figures $5 \mathrm{~B}$ and $6 \mathrm{~B}$ ). Of note, newly formed blood vessels escaping the inhibitory effects of anti-IL-8 treatment did not exhibit enhanced pericyte coverage, which remained sparse and, therefore, could not have accounted for increased microcirculation leading to similar growth rates of primary tumors despite decreased levels of intratumoral angiogenesis (see Supplemental Figure S6 at http://ajp.amjpathol.org). A coordinated $40 \%$ to $50 \%$ dimin- 
A

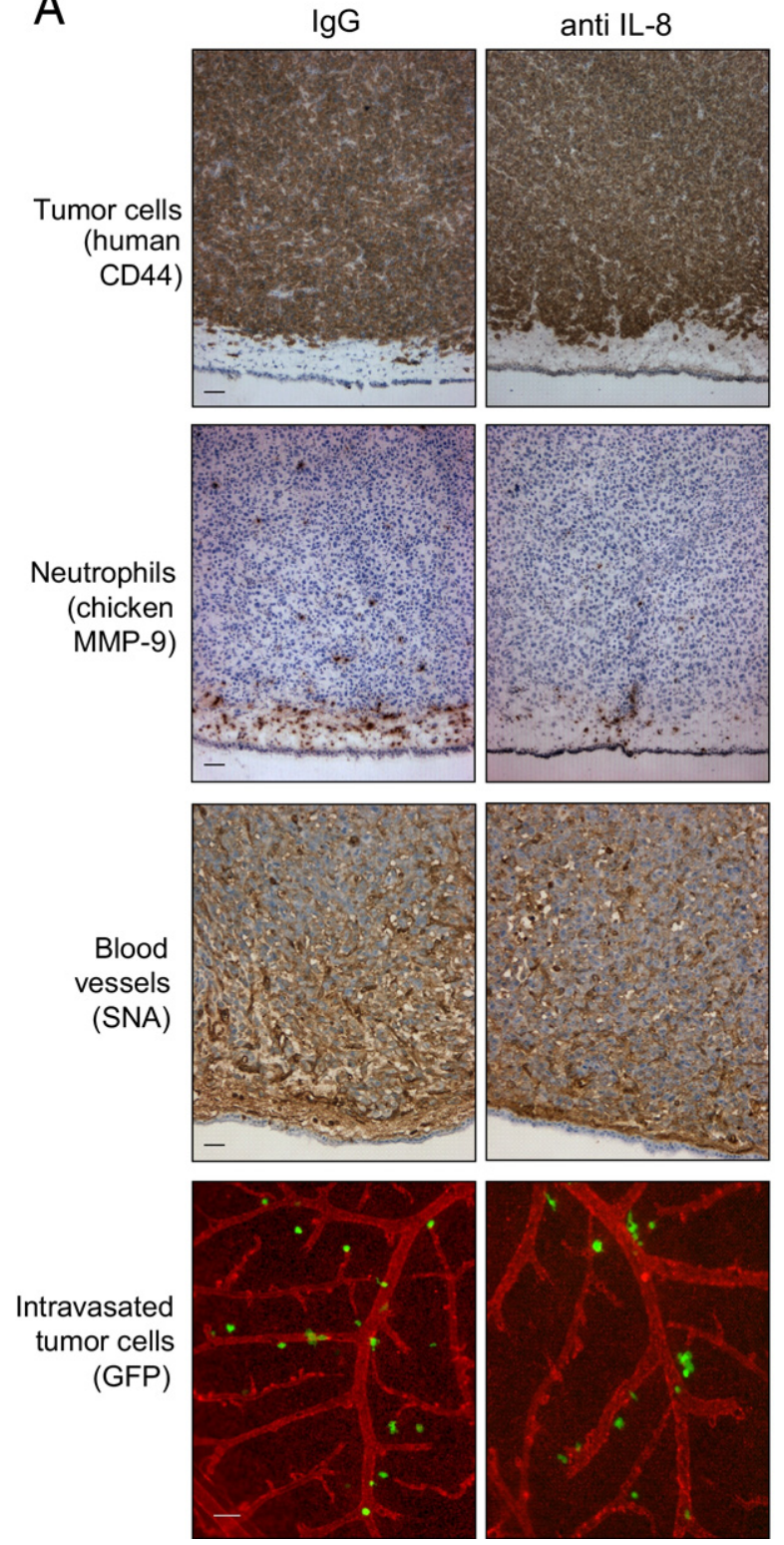

B
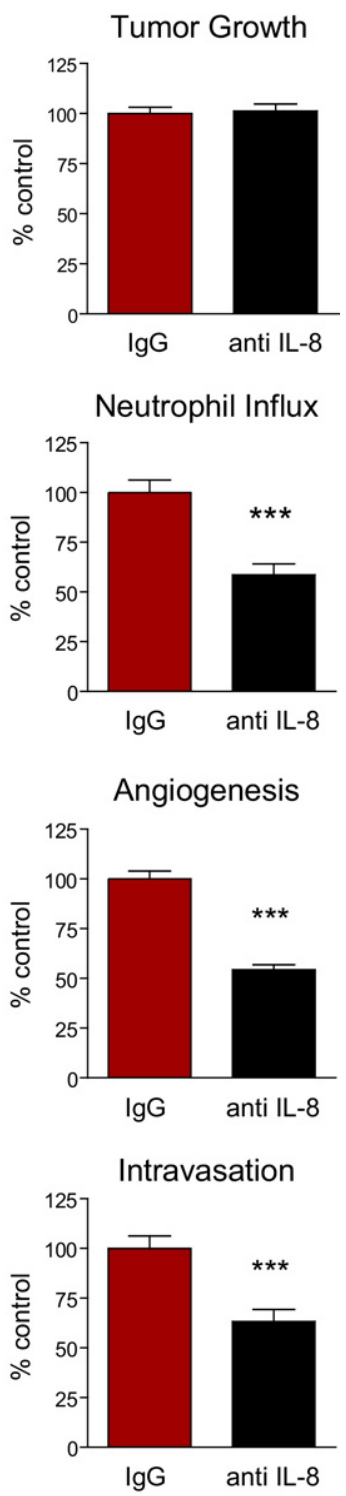

Figure 5. Specific inhibition of neutrophil influx into HT-hi/diss primary tumors coordinately diminishes tumor angiogenesis and tumor cell intravasation. HT-hi/diss primary tumors were treated with $20 \mu \mathrm{g}$ control IgG or IL-8/CXCL8neutralizing antibody (anti-IL-8) in five independent experiments. A: Immunohistochemical analysis of primary tumors. Sections from four to six individual tumors were stained with antihuman CD44 to discriminate human tumor cells (brown), anti-chicken MMP-9 antibody to visualize MMP-9-positive neutrophils (brown), and SNA to highlight blood vessels (brown). Intravasated GFP-tagged HT-hi/diss cells (green) were visualized using live cell imaging in the CAM in which blood vessels were highlighted by red fluorescent LCA. Scale bars $=50 \mu \mathrm{m}$. B: Tumor growth, neutrophil influx, tumor angiogenesis, and intravasation were quantified as described in Materials and Methods. Sixty-one and 47 tumor-bearing embryos were analyzed in control IgG- and anti IL-8-treated groups, respectively. Data are expressed as a percentage of IgG control and represent mean \pm SEM. ${ }^{* * * *} P<0.001$ two-tailed Student's $t$-test. ishment in neutrophil recruitment and tumor angiogenesis, caused by IL-8 neutralization, was accompanied by a corresponding reduction in HT-hi/diss and PC-hi/diss tumor cell intravasation by $40 \%$ and $45 \%$ of IgG controls, respectively (Figures 5B and 6B). Therefore, these findings functionally link neutrophil influx with tumor angiogenesis and intravasation in two highly metastatic variants of human cancer cells of different tissue origin: mesenchymal fibrosarcoma and epithelial carcinoma.

\section{Functional and Mechanistic Contribution of Neutrophil TIMP-Free proMMP-9 to Tumor Cell Intravasation via Angiogenic Blood Vessels}

It was hypothesized that by specifically rescuing dampened neutrophil influx with purified neutrophil proMMP-9, we would show directly that proMMP-9 naturally devoid of
TIMP-1 can functionally contribute to both tumor angiogenesis and intravasation. Thus, a highly purified fraction of MMP-9-containing neutrophils was isolated from human peripheral blood (see Supplemental Figure S7 at http://ajp.amjpathol.org), induced to release their secretory granules, and neutrophil TIMP-free proMMP-9 was purified using affinity chromatography. A portion of the preparation was stoichiometrically complexed with recombinant TIMP-1 and repurified (Figure 7A). The use of this proMMP-9-TIMP-1 complex allowed us to confirm that the TIMP-free status of neutrophil proMMP-9 is a critical biochemical condition rendering this readily activatable form of MMP-9 zymogen with high potency not only during physiologic angiogenesis ${ }^{29,31}$ but also during tumor-induced angiogenesis. In parallel, neutrophil proMMP-9 was used in a mixture with a molar excess of TIMP-2, therefore making it possible to validate whether 
A

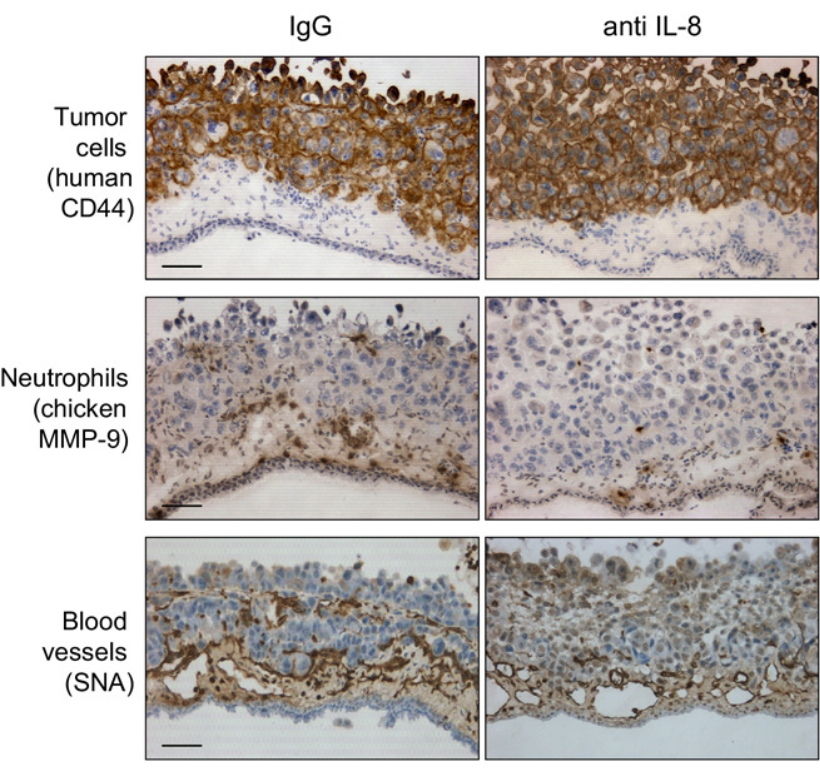

B
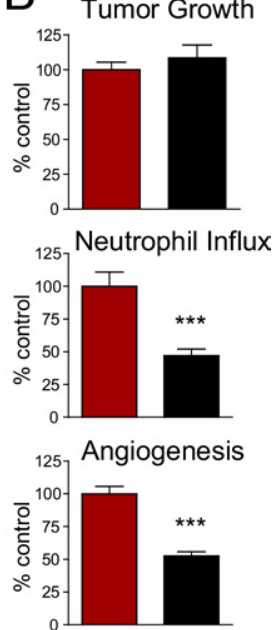

Intravasation

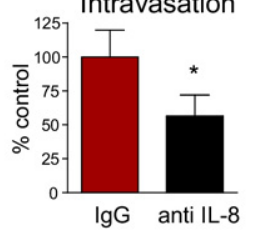

Figure 6. Specific inhibition of neutrophil in flux into PC-hi/diss primary tumors coordinately diminishes tumor angiogenesis and tumor cell intravasation. PC-hi/diss primary tumors were treated with $20 \mu \mathrm{g}$ control IgG or IL-8/CXCL8neutralizing antibody (anti IL-8) in three independent experiments. A: Immunohistochemical analysis of PC-hi/diss primary tumors. Sections from four to six individual tumors were stained with anti-human CD44 to differentiate human tumor cells (brown), anti-chicken MMP-9 antibody to visualize MMP-9-positive neutrophils (brown), and SNA to highlight blood vessels (brown). Original magnification, $\times 200$. Scale bars $=50 \mu \mathrm{m}$. B: Tumor growth, neutrophil influx, tumor angiogenesis, and intravasation were quantified as described in Materials and Methods. Thirty and 22 tumor-bearing embryos were analyzed in control IgG- and anti IL-8treated groups, respectively. Data are expressec as a percentage of $\operatorname{IgG}$ control and represent mean \pm SEM. ${ }^{*} P<0.05$, and ${ }^{* * * * *} P<0.001$ two-tailed Student's $t$-test. the resulting proteolytic activity of neutrophil MMP-9 enzyme is functionally involved in tumor-induced angiogenesis.

These described suggestions were first verified in the CAM angiogenesis model, in which the angiogenesis induced by HT-hi/diss cells incorporated into 3D collagen rafts was significantly inhibited by IL-8-neutralizing antibody. The inhibitory effects of anti-IL-8 treatment were reversed by addition of TIMP-free neutrophil proMMP-9, but not if proMMP-9 was pre-complexed with TIMP-1 or was used in a mixture with TIMP-2 (Figure 7B). These findings provided a solid background for experiments in a more complex setting in which angiogenesis was analyzed in developing primary tumors in conjunction with tumor cell intravasation (Figure 7, C and D). The developing HT-hi/diss tumors were treated with anti-IL-8 antibody with or without nanogram quantities of TIMP-free neutrophil proMMP-9 or neutrophil proMMP-9 either as a stoichiometric complex with TIMP-1 or mixed with TIMP-2. Supplementation of proMMP-9 did not rescue neutrophil recruitment that was inhibited by anti-IL-8 treatment (data not shown), consistent with the notion that although being a potent angiogenic protease, proMMP-9 does not act as a direct neutrophil attractant. However, exogenously added TIMP-free neutrophil proMMP-9 coordinately restored both tumor angiogenesis and intravasation, which were diminished by anti-IL-8 therapy. In contrast, the levels of HT-hi/diss angiogenesis and intravasation, dampened by IL-8 neutralization, were not restored when the stoichiometric complex between neutrophil proMMP-9 and TIMP-1 was added to CAM tumors or if TIMP-2 was applied along with TIMP-1-free neutrophil proMMP-9 (Figure 7, C and D). These findings strongly implicate the natural TIMP-free status of neutrophil MMP-9 proenzyme and the proteolytic activity of neutro- phil MMP-9 enzyme in the contribution of inflammatory neutrophils to both tumor angiogenesis and tumor cell intravasation. Finally, significant differentials in the levels of intratumoral angiogenesis were not accompanied by substantial changes in weight of HT-hi/diss tumors (Figure 7D) or their histologic composition (see Supplemental Figure S8 at http://ajp.amjpathol.org), making it possible to directly link the levels of tumor angiogenesis and tumor cell intravasation.

\section{Discussion}

Increasing experimental evidence has implicated infiltrating neutrophils and neutrophil MMP-9 in the synchronized promotion of tumor angiogenesis and primary tumor growth. ${ }^{18,20,38,43,44}$ However, to our knowledge, there are no reports demonstrating that inflammatory neutrophils import MMP-9 and facilitate in a coordinated fashion tumor angiogenesis and spontaneous tumor cell dissemination. The objective of the present study, therefore, was to mechanistically link tumor-infiltrating neutrophils and neutrophil MMP-9 with tumor angiogenesis and tumor cell intravasation. Neutrophil-derived proMMP-9 is distinctive because it is synthesized and released in a TIMP-free form, ${ }^{45}$ which, as we have shown previously, renders it highly activatable and with an unprecedented potency to trigger in vivo tumor-free physiologic angiogenesis. ${ }^{29,31}$ Subnanomolar concentrations of TIMP-free MMP-9, in sharp contrast to the TIMP-encumbered form, are sufficient to induce physiologic angiogenesis, which suggests that TIMP-free status is a critical molecular determinant of MMP-9 released by neutrophils.

A number of studies have correlated elevated levels of neutrophils in patient samples with increased tumor inva- 
A

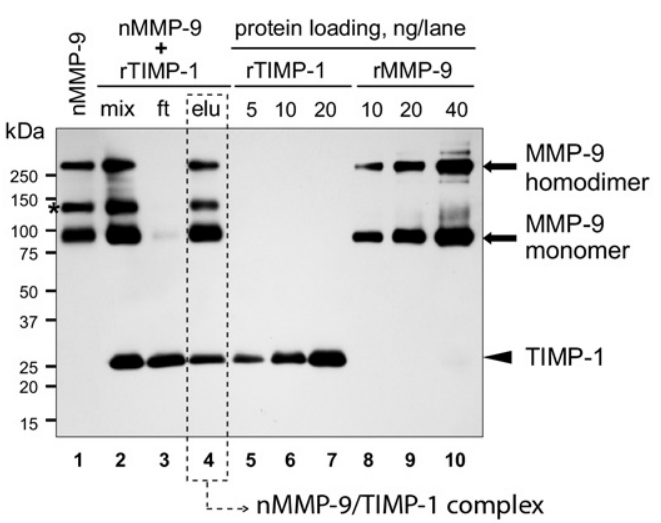

C

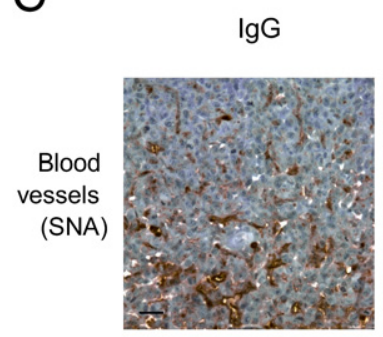

Tumor Growth

D

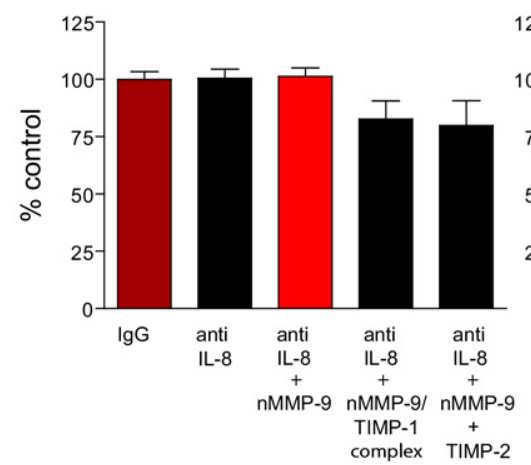

B

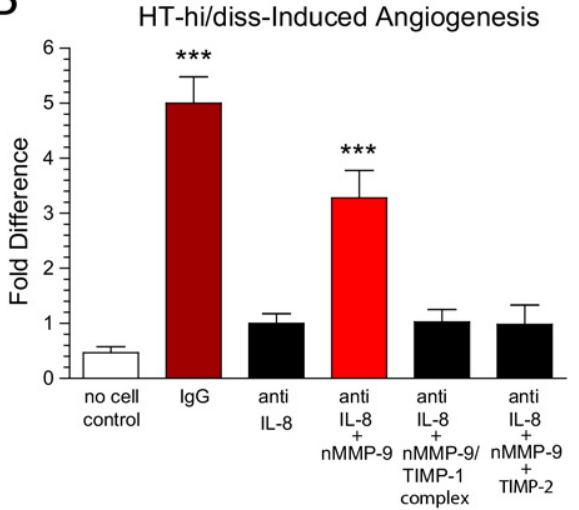

anti IL-8 + nMMP-9

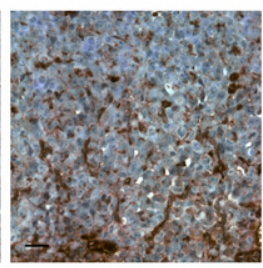

Tumor Angiogenesis anti IL-8 + nMMP-9/TIMP-1 complex

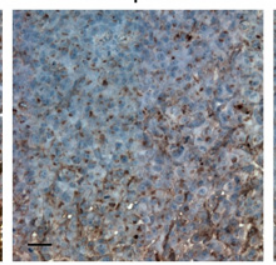

anti IL-8

+ nMMP-9

+ TIMP-2

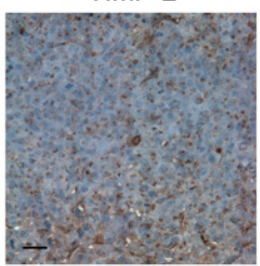

Tumor Cell Intravasation

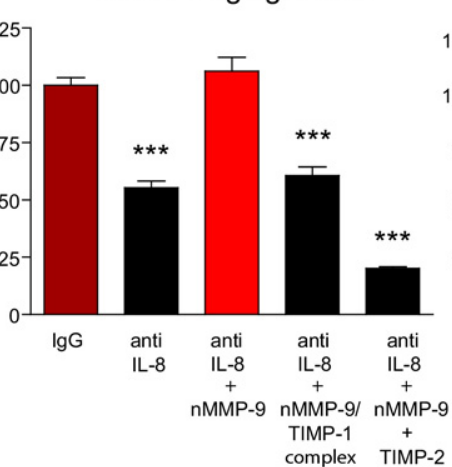

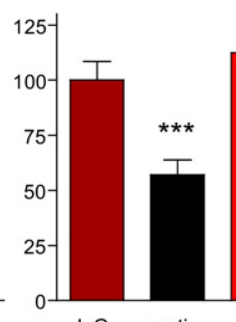

$\lg G$

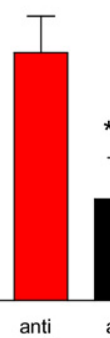

anti
$\mathrm{IL}-8$
+
$\mathrm{nMMP}$

nMMP-

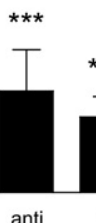

anti anti

$\mathrm{IL}-8 \quad \mathrm{IL}-8$

$\stackrel{+}{+} \stackrel{+}{+}$

TIMP-1 +

Figure 7. Rescue of diminished tumor angiogenesis and tumor cell intravasation via delivery of exogenous TIMP-free neutrophil promMP-9. A: Generation of neutrophil proMMP-9-TIMP-1 complex. Neutrophils isolated from human peripheral blood were induced to release their MMP-9-containing secretory granules, and their proMMP-9 was purified using affinity chromatography (nMMP-9, lane 1). To generate the neutrophil proMMP-9-TIMP-1 complex, purified neutrophil proMMP-9 was mixed with recombinant human TIMP-1 (rTIMP-1) at 1:4 molar ratio (mix, lane 2). After incubation for 1 hour at ambient temperature, the protein mixture (mix) was applied to gelatin Sepharose beads to recover the formed proMMP-9-TIMP-1 complex (elu, lane 4) and remove excess TIMP-1 in the flow-through fraction (ft, lane 3). The proteins were separated using SDS-PAGE under nonreducing conditions, and Western immunoblot analysis was performed using a mixture of mouse anti-human MMP-9 and anti-TIMP-1 mAbs. Lanes 5-7 and 8-10: Loading controls for recombinant TIMP-1 and recombinant proMMP-9, respectively (nanograms per lane). The positions of molecular weight standards are indicated in kilodaltons on the left. Asterisk indicates the position of a $125-\mathrm{kDa}$ heterodimer between proMMP-9 and NGAL (neutrophil gelatinase-associated lipocalin), unique to neutrophils. The molar amounts of proMMP- 9 ( 92 $\mathrm{kDa})$ and TIMP-1 (28 kDa) eluted from gelatin Sepharose beads (dashed box) were calculated via comparison with corresponding protein loading controls and indicated a 1:1 stoichiometric complex between proMMP-9 and TIMP-1. B: Angiogenic and rescuing potential of neutrophil MMP-9 in the chick embryo collagen onplant model. HT-hi/diss cells were incorporated into 3D collagen onplants at $1 \times 10^{6}$ cells $/ \mathrm{mL}$ with $3 \mu \mathrm{g} / \mathrm{mL}$ normal IgG or IL-8/CXCL8-neutralizing antibody (anti IL-8). Control onplants contained collagen only (no cell control). A subset of the HT-hi/diss-containing collagen onplants treated with anti IL-8 were additionally supplemented with $2 \mathrm{ng}$ purified neutrophil proMMP-9 (nMMP-9) or a stoichiometric 1:1 molar complex between neutrophil proMMP-9 and TIMP1 corresponding to 2 ng nMMP-9 (nMMP-9-TIMP-1) or 2 ng neutrophil proMMP-9 mixed with 5 ng TIMP-2 (eightfold molar excess over nMMP-9). In two independent experiments, 22 to 42 individual onplants were analyzed in each group. Data are expressed as fold difference \pm SEM calculated over angiogenesis levels in the anti IL- 8 group. ${ }^{*} *{ }^{*} P<0.001$, two-tailed Student's $t$-test. C and D: Angiogenic and rescuing capacity of neutrophil MMP-9 in the chick embryo spontaneous metastasis model. HT-hi/diss primary tumors, developing on the CAM, were treated with control IgG or IL-8/CXCL8 neutralizing antibody (anti IL-8) in three independent experiments. A subset of anti-IL-8-treated tumors were additionally supplemented with 30 ng nMMP-9, purified nMMP-9/TIMP-1 stoichiometric complex corresponding to $30 \mathrm{ng}$ nMMP-9, or a mixture of $30 \mathrm{ng}$ nMMP-9 and 70 ng TIMP-2 (7.7-fold molar excess over nMMP-9). C: Immunohistochemical analysis of angiogenesis in primary tumors was performed on tissue sections stained with SNA to highlight blood vessels (brown). Scale bars $=25 \mu \mathrm{m}$. D: Tumor growth, angiogenesis, and intravasation were quantified in three independent experiments involving up to 45 tumor-bearing embryos for each treatment condition. Data are expressed as a percentage of IgG control and represent mean \pm SEM. ${ }^{\text {*** }} P<0.001$, two-tailed Student's $t$-test.

sion and poor prognosis in several types of cancer. ${ }^{8,21,26}$ An increase in neutrophil infiltration has been demonstrated in colorectal cancer progression, eg, from premalignant aberrant crypt foci to adenomas to carcinomas. ${ }^{46}$
Steadily emerging experimental evidence also directly indicates that tumor-recruited neutrophils and neutrophil MMP-9 positively contribute to tumor angiogenesis and tumor development early in cancer progression. Thus, in 
a transgenic mouse model of multistage pancreatic tumorigenesis, the specific contribution of tumor-infiltrating neutrophils to tumor angiogenesis was demonstrated at the early neoplastic stage but not at later stages of tumor progression. ${ }^{47}$ In the same model, MMP-9-positive neutrophils infiltrated the premalignant islet dysplasias, and neutrophil-delivered MMP-9 specifically triggered the angiogenic switch, ${ }^{18}$ in contrast to a previously ascribed angiogenic function of macrophage-derived MMP-9. ${ }^{11}$ In mice lacking the CCR2 macrophage receptor, which is required for tissue recruitment of monocytes, MMP-9 positive neutrophils can become the dominant tumorinfiltrating leukocyte type promoting tumor growth and tumor angiogenesis. ${ }^{48}$ That tumor-associated neutrophils represent a critical cell constituent within the tumor tissue microenvironment was also demonstrated when the suppressed recruitment of tumor-promoting macrophages to the tumor was compensated for by enhanced infiltration of MMP-9-positive neutrophils. ${ }^{20}$

These cited studies and our data presented herein do not imply that tumor-influxing neutrophils function exclusively or manifest a dominant role compared with tumorassociated macrophages in promoting cancer progression. Rather, these findings suggest that neutrophils can cooperate with monocytes, macrophages, and other infiltrating bone marrow-derived myeloid cells in delivering, possibly at different times, critical effector molecules into the developing tumor tissue. Depending on their cellular source, the contributing molecules can vary in nature and functional potency. Taking into account the now well-documented links between MMP-9 and development of premetastatic niches ${ }^{4,5}$ and MMP-9 and the induction of tumor angiogenesis, ${ }^{11,12,18,20}$ we propose that mature neutrophils immediately or their granulocytic precursor cells eventually are best suited to provide the most potent, efficient, and concentrated form of a functional MMP-9 molecule.

To demonstrate that tumor-infiltrating neutrophils and neutrophil MMP-9 not only contribute to tumor angiogenesis but also synchronously enhance hematogenous dissemination, we used variants of human tumor cells selected from parental HT-1080 fibrosarcoma and PC-3 prostate carcinoma for enhanced levels of intravasation and dissemination. Highly disseminating variants, ie, HThi/diss and PC-hi/diss, were compared with their lo/diss counterparts insofar as their ability to intravasate and metastasize, induce angiogenesis, and recruit MMP-9producing neutrophils. Furthermore, the critical aspects of hi/diss versus lo/diss tumor cell pathophysiologic features were reproduced in corresponding mammalian xenograft models, ie, heterotopic implantation under the kidney capsule (Figure 1) or orthotopic implantation into the prostate gland, ${ }^{36}$ thereby affirming that our selection for distinct malignant characteristics was not limited to avian models. The results of these comparative analyses indicated that the high propensity of the aggressive tumor cells to intravasate correlated positively and strongly with their high angiogenic potential and ability to recruit MMP-9-positive neutrophils. Furthermore, the exogenous addition of proMMP-9 isolated from human neutro- phils efficiently enhanced the angiogenic potential of the nonaggressive lo/diss cells of both cancer types.

That neutrophil MMP-9 can increase the angiogenic potential of both fibrosarcoma and prostate carcinoma lo/diss cells indicated that the enhanced recruitment of MMP-9-delivering neutrophils into hi/diss tumors might be an important functional characteristic of aggressive tumor cells with high angiogenic potential. This suggestion was confirmed in the collagen onplant model by the use of an anti-inflammatory drug, ibuprofen, which significantly decreased angiogenesis induced by HT-hi/diss and PC-hi/diss cells. Ibuprofen-inhibited angiogenesis was completely restored by exogenously supplemented neutrophil releasate containing proMMP-9 or by TIMPfree proMMP-9 purified from isolated neutrophils. Because this critical angiogenesis-restoring ability was lost in the neutrophil releasates that were specifically depleted of proMMP-9, our findings demonstrate that the angiogenic potential of these aggressive tumor cells is linked to their ability to attract MMP-9-delivering neutrophils to primary tumors.

To demonstrate directly that recruited neutrophils supply their unique proMMP-9 and determine both the levels of tumor angiogenesis and dissemination, we designed a series of experiments to prevent specifically the influx of neutrophils into developing primary tumors. This neutrophil-specific inhibition was achieved by neutralizing human IL-8 (CXCL8), a potent mediator of neutrophil recruitment. ${ }^{40,41}$ Although the levels of IL-8 produced by hi/diss and lo/diss counterparts are similar, and, therefore, IL-8 expression alone is unlikely to contribute to their intravasation differential, it is possible that IL-8 produced by hi/diss cells in vivo is functionally more active due to its proteolytic processing. Indeed, neutrophil MMP-9 has been demonstrated to potentiate the functional activity of IL-8 from 10- to 30 -fold, ${ }^{49}$ which would implicate a positive feedback loop for hi/diss tumor variants that exhibit high levels of neutrophil attraction. Alternatively, the lo/diss tumors may produce inhibitors of neutrophil influx in vivo or inhibitors of IL-8 processing, thereby accounting for lower levels of inflammatory cell influx even in the presence of IL-8 at levels similar to those produced by hi/diss tumors.

Taking all of these considerations into account, we used IL-8 neutralization to specifically inhibit neutrophil infiltration into hi/diss tumors to analyze the contributions of influxing neutrophils and neutrophil MMP-9 in tumor angiogenesis and intravasation. The inhibitory effects of neutralizing IL-8 on tumor-induced angiogenesis were first evidenced in our CAM angiogenesis model measuring the angiogenic potential of tumor cells embedded into 3D collagen grafts. Importantly, the levels of HT-hi/ diss-induced angiogenesis, which had been diminished by anti-IL-8 therapy, were rescued by the addition of neutrophil TIMP-free proMMP-9. The lack of such reversal in angiogenesis if neutrophil proMMP-9 was complexed with TIMP-1 or used along with TIMP-2 strongly indicated that both activation of the MMP-9 zymogen, delivered by inflammatory neutrophils, and the activity of activated neutrophil MMP-9 enzyme functionally contribute to the angiogenic response induced by HT-hi/diss cells. 
In the more complex model system in which metastasizing primary tumors develop within the CAM tissue, histochemical analysis of HT-hi/diss and PC-hi/diss tumors confirmed that anti IL-8 therapy resulted in a significant reduction in the influx of MMP-9-positive neutrophils concomitant with reduced levels of hi/diss tumor angiogenesis and intravasation. In contrast, IL-8-neutralizing antibody had little or no effect on the influx of tumor-recruited monocytes/macrophages and primary tumor growth. Therefore, the specific prevention of neutrophil infiltration into developing primary tumors by blocking the IL-8/CXCR1 chemotactic pathway clearly linked the diminished levels of inflammatory neutrophils with subsequent inhibition of tumor angiogenesis and tumor cell intravasation.

Because neutrophils are a major cellular source of MMP-9 in the chick embryo, ${ }^{39}$ replenishing the MMP-9 deficit, caused by reduced neutrophil influx, with purified neutrophil proMMP-9 enabled us to specifically address whether neutrophil MMP-9 regulates the levels of angiogenesis in primary tumors and dissemination of malignant cells from primary tumors. The clear rescue of antiIL-8 inhibitory effects on tumor angiogenesis by exogenous delivery of neutrophil TIMP-free proMMP-9 but not by the neutrophil proMMP-9 complexed with TIMP-1 or mixed with TIMP-2 demonstrated that both the activation of MMP-9 proenzyme and the enzymatic activity of activated proMMP-9 are critically involved in angiogenesis-dependent spread of malignant tumor cells. These findings also indicate that the high local concentration of the unique form of proMMP-9 released by tumor-recruited neutrophils is essential for tumor angiogenesis and tumor cell intravasation. Therefore, the coordinated rescue of damped tumor angiogenesis and tumor cell intravasation by exogenous delivery of neutrophil proMMP-9 strongly support the contribution of tumorrecruited neutrophils and their TIMP-free MMP-9 to overall tumor progression.
Inhibition of angiogenesis usually results in decreased tumor growth and metastasis in long-term mammalian metastasis models, and, therefore, it is often not possible to dissociate smaller tumor burden from coordinately decreased tumor dissemination. It is precisely this direct correlation between angiogenesis levels and tumor size in murine models that does not enable an unequivocal conclusion as to whether inhibitory effects of anti-cancer treatments on tumor cell dissemination were achieved due to inhibition of angiogenesis and angiogenesis-dependent processes (eg, intravasation) or simply to suppression of tumor growth and the corresponding decrease in the number of tumor cells capable of dissemination. In contrast, in the chick embryo model of metastasis, primary tumors develop within the CAM tissue, which contains a dense vascular network of capillaries, comparable in density to the lungs of adult mice, thereby providing a continuous supply of nutrients and efficient gas exchange even when intratumor angiogenesis is suppressed. This unique feature enables development of tumors similar in size despite substantial differences in the levels of intratumoral angiogenesis. Moreover, high levels of gas exchange and nutrient supply at all stages of CAM tumor development almost completely negate the hypoxia-, necrosis- and apoptosis-induced effects of angiogenesis deficiency that are commonly observed in murine xenograft models undergoing therapeutic targeting of tumor angiogenesis. Therefore, despite differences in the levels of tumor angiogenesis, primary CAM tumors may not differ substantially in net weight and histologic appearance. In addition, such compensatory mechanisms as "normalization" of blood vessels escaping antiangiogenic treatments, reflected in enhanced pericyte coverage of tumor vasculature, may not have as critical a role in developing primary CAM tumors as in mammalian tumors, in which improved microcirculation, reflected in enhanced vascular perfusion, can account for similar or even enhanced rates of tumor growth and dissemination.

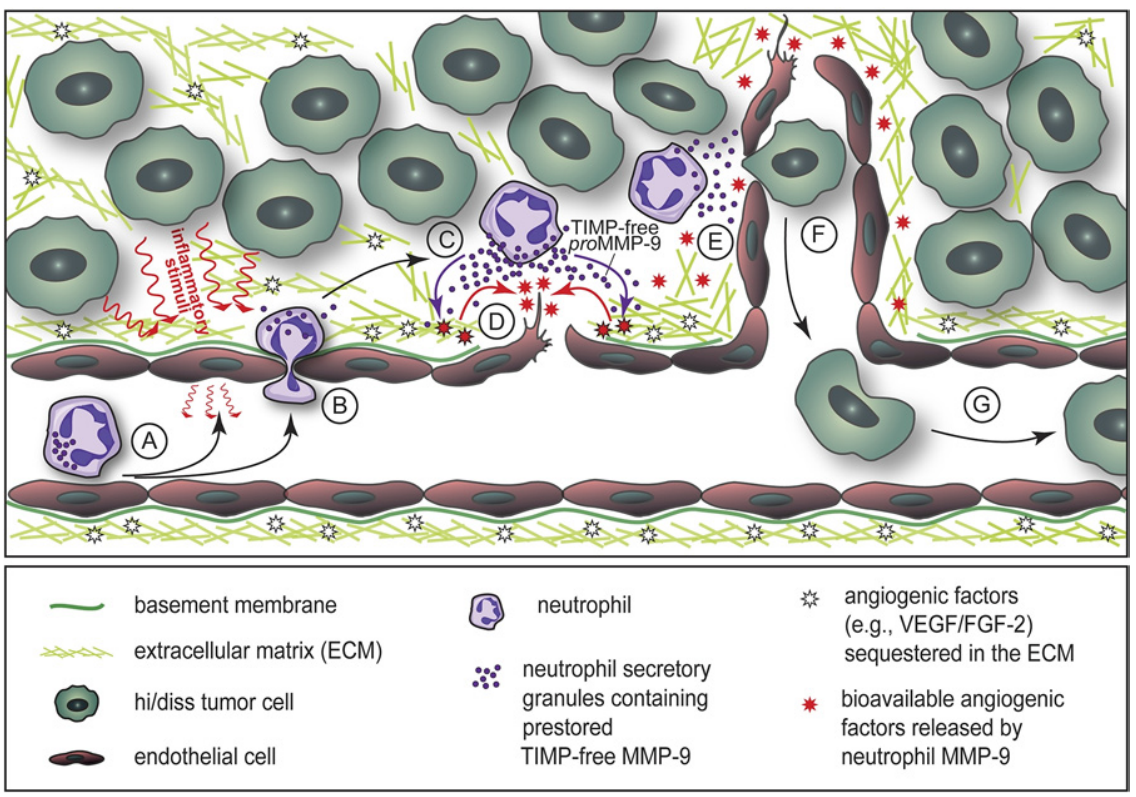

Figure 8. The functional role of neutrophils and their unique TIMP-free MMP-9 in tumor angiogenesis and tumor cell intravasation. Schema demonstrates the attraction of circulating neutrophils to the luminal surface endothelial cells activated by inflammatory stimuli produced by tumor and/or stromal cells (A), followed by neutrophil extravasation into tumor stroma (B), release of secretory granules containing prestored TIMP-free proMMP-9 from induced neutrophils (C), activation of neutrophil proMMP-9 by as yet undefined proteolytic mechanisms to produce the active MMP-9 enzyme catalytically capable of remodeling the ECM and the efficient release of ECM-sequestered angiogenic factors such as VEGF and FGF-2 (D), which in turn induce endothelial cell sprouting and formation of new blood vessels $(\mathbf{E})$, intravasation of tumor cells, likely at specific entry points where ECM and endothelial basement membrane have been proteolytically modified by neutrophil MMP-9 enzyme (F), and dissemination of intravasated tumor cells via circulation $(\mathbf{G})$. 
The human tumor and CAM xenograft models thus eliminate a number of confounding indirect effects of angiogenesis inhibition that could positively or negatively affect tumor dissemination. Therefore, the findings of the present study, demonstrating that tumor cell dissemination positively and coordinately correlates with actual levels of angiogenesis in primary tumors, independently of the primary tumor burden, provide a strong argument for the notion that the newly formed angiogenic vessels offer ample entry points for tumor cell intravasation and serve as conduits for tumor cell dissemination.

It should be emphasized that although this study focused on the role of MMP-9-delivering neutrophils in both tumor angiogenesis and intravasation, the findings do not exclude the contribution to these processes of inflammatory CD11b-, Gr-1-, or Ly6C-positive immature myeloid cells or monocytes/ macrophages, which have long been reported to contribute critical molecules and enhance tumor progression and metastasis. ${ }^{6,7,9,21,23-25}$ However, a direct evaluation of CD11b- and Gr1-positive cells isolated from tumors of different histologic types indicated low levels of MMP-9 expression by tumor-associated $\mathrm{CD} 11 \mathrm{~b}^{+} \mathrm{Gr} 1^{\text {int/dul }}$ Ly6 $\mathrm{C}^{\text {hi }}$ macrophages versus $\mathrm{CD} 11 \mathrm{~b}^{+} \mathrm{Gr} 1^{\text {hi }}$ granulocytes. ${ }^{48}$ Multimodal analysis of cellular sources of MMP-9 in patients with colon adenocarcinomas demonstrated that only tumor-associated neutrophils were routinely positive for high levels of MMP-9 protein, whereas MMP-9 mRNA-positive macrophages expressed MMP-9 protein in amounts below the detection limit of immunohistochemistry. ${ }^{50}$ In addition, we have previously demonstrated that monocytic cells produce proMMP-9 stoichiometrically complexed with TIMP-1, which prevents its efficient proteolytic activation and renders monocytic MMP-9 inefficient in induction of physiologic angiogenesis relative to neutrophil TIMP-free proMMP-9. ${ }^{31}$ Therefore, neutrophils, with their high responsiveness to cytokines, early arrival in the tumor stromal tissue, and rapid release of prestored readily activatable proMMP-9 at high local concentrations, are far better equipped to initiate the angiogenic switch.

In addition to the proteolytic activity of MMPs, and neutrophil MMP-9 in particular, the involvement of non-MMP proteases such as urokinase-type plasminogen activator (UPA) and plasmin in tumor angiogenesis and metastasis has been demonstrated previously in our model systems. By using activation-blocking anti-uPA mAb-112 and aprotinin, which inhibit pro-uPA activation and the activity of UPA-generated plasmin, respectively, we demonstrated the functional contribution of these two serine proteases in tumor cell-induced angiogenesis, Matrigel invasion, and intravasation of both HT-hi/diss ${ }^{51,52}$ and PC-hi/diss. ${ }^{36}$ Our most recent findings specifically indicate that the UPA/ plasmin system has a critical role in the earlier events of the metastatic cascade such as invasive escape from the primary PC-hi/diss tumors (Bekes et al, unpublished observations). Therefore, it seems that both MMP-dependent and serine protease-dependent mechanisms are involved in early metastatic events, and, therefore, independent inhibition of either of these systems most probably would not be enough to drastically decrease or eradicate tumor cell dissemination.
Our view of a possible pivotal role of inflammatory neutrophils delivering a unique form of MMP-9 to regulate the levels of tumor angiogenesis and tumor cell intravasation is presented schematically in Figure 8. Starting with the attraction of circulating neutrophils to the luminal surface of endothelial cells, which could have been activated by various inflammatory stimuli produced by tumor and/or stromal cells (Figure 8A), the schema depicts further critical steps including neutrophil extravasation (Figure 8B), neutrophil induction and release of secretory granules containing TIMP-free proMMP-9 (Figure 8C), activation of neutrophil proMMP-9 by as yet undefined mechanisms to produce the active MMP-9 enzyme, now catalytically capable of efficient release of ECM-sequestered angiogenic factors such as VEGF and FGF-2 (Figure $8 D$ ), which in turn induce endothelial cell sprouting and formation of new blood vessels (Figure 8E), serving as conduits for neutrophil and neutrophil MMP-9-assisted tumor cell intravasation (Figure 8F) and dissemination (Figure 8G). Collectively, the findings of the present study enabled us for the first time to directly link the ability of aggressive tumor cells to intravasate with their ability to recruit inflammatory neutrophils delivering their unique TIMP-free proMMP-9, which in turn rapidly and potently induces tumor angiogenesis, thereby providing the vascular conduits for tumor cell dissemination.

\section{Acknowledgments}

We thank Dr. Rafael Fridman for generously providing recombinant TIMP-1 and TIMP-2, Chenxing Li for expert technical assistance, and Dr. Ghislain Opdenakker for his support of this study and valuable discussions.

\section{References}

1. Coussens LM, Werb Z: Inflammation and cancer. Nature 2002, 420: 860-867

2. Joyce JA: Therapeutic targeting of the tumor microenvironment. Cancer Cell 2005, 7:513-520

3. Mantovani A, Allavena P, Sica A, Balkwill F: Cancer-related inflammation. Nature 2008, 454:436-444

4. Wels J, Kaplan RN, Rafii S, Lyden D: Migratory neighbors and distant invaders: tumor-associated niche cells. Genes Dev 2008, 22:559-574

5. Psaila B, Lyden D: The metastatic niche: adapting the foreign soil. Nat Rev Cancer 2009, 9:285-293

6. Shojaei F, Zhong C, Wu X, Yu L, Ferrara N: Role of myeloid cells in tumor angiogenesis and growth. Trends Cell Biol 2008, 18:372-378

7. Solinas G, Germano G, Mantovani A, Allavena P: Tumor-associated macrophages (TAM) as major players of the cancer-related inflammation. J Leukoc Biol 2009, 86:1065-1073

8. Tazzyman S, Lewis CE, Murdoch C: Neutrophils: key mediators of tumour angiogenesis. Int J Exp Pathol 2009, 90:222-231

9. Coffelt SB, Lewis CE, Naldini L, Brown JM, Ferrara N, De Palma M Elusive identities and overlapping phenotypes of proangiogenic myeloid cells in tumors. Am J Pathol 2010, 176:1564-1576

10. Gregory AD, McGarry Houghton A: Tumor-associated neutrophils: new targets for cancer therapy. Cancer Res 2011, 71:2411-2416

11. Bergers G, Brekken R, McMahon G, Vu TH, Itoh T, Tamaki K, Tanzawa K, Thorpe P, Itohara S, Werb Z, Hanahan D: Matrix metalloproteinase- 9 triggers the angiogenic switch during carcinogenesis. Nat Cell Biol 2000, 2:737-744

12. Coussens LM, Tinkle CL, Hanahan D, Werb Z: MMP-9 supplied by bone marrow-derived cells contributes to skin carcinogenesis. Cell 2000, 103:481-490 
13. Huang S, Van Arsdall M, Tedjarati S, McCarty M, Wu W, Langley R, Fidler IJ: Contributions of stromal metalloproteinase-9 to angiogenesis and growth of human ovarian carcinoma in mice. J Natl Cancer Inst 2002, 94:1134-1142

14. Giraudo E, Inoue M, Hanahan D: An amino-bisphosphonate targets MMP-9-expressing macrophages and angiogenesis to impair cervical carcinogenesis. J Clin Invest 2004, 114:623-633

15. Jodele S, Chantrain CF, Blavier L, Lutzko C, Crooks GM, Shimada H, Coussens LM, Declerck YA: The contribution of bone marrowderived cells to the tumor vasculature in neuroblastoma is matrix metalloproteinase-9 dependent. Cancer Res 2005, 65:3200-3208

16. Acuff HB, Carter KJ, Fingleton B, Gorden DL, Matrisian LM: Matrix metalloproteinase- 9 from bone marrow-derived cells contributes to survival but not growth of tumor cells in the lung microenvironment. Cancer Res 2006, 66:259-266

17. Chantrain CF, Henriet P, Jodele S, Emonard H, Feron O, Courtoy PJ, DeClerck YA, Marbaix E: Mechanisms of pericyte recruitment in tumour angiogenesis: a new role for metalloproteinases. Eur J Cancer 2006, 42:310-318

18. Nozawa H, Chiu C, Hanahan D: Infiltrating neutrophils mediate the initial angiogenic switch in a mouse model of multistage carcinogenesis. Proc Natl Acad Sci USA 2006, 103:12493-12498

19. Du R, Lu KV, Petritsch C, Liu P, Ganss R, Passegue E, Song H, Vandenberg S, Johnson RS, Werb Z, Bergers G: HIF1alpha induces the recruitment of bone marrow-derived vascular modulatory cells to regulate tumor angiogenesis and invasion. Cancer Cell 2008, 13:206-220

20. Pahler JC, Tazzyman S, Erez N, Chen YY, Murdoch C, Nozawa H, Lewis CE, Hanahan D: Plasticity in tumor-promoting inflammation: impairment of macrophage recruitment evokes a compensatory neutrophil response. Neoplasia 2008, 10:329-340

21. Murdoch C, Muthana M, Coffelt SB, Lewis CE: The role of myeloid cells in the promotion of tumour angiogenesis. Nat Rev Cancer 2008 8:618-631

22. Kessenbrock K, Plaks V, Werb Z: Matrix metalloproteinases: regulators of the tumor microenvironment. Cell 2010, 141:52-67

23. Pollard JW: Tumour-educated macrophages promote tumour progression and metastasis. Nat Rev Cancer 2004, 4:71-78

24. Condeelis J, Pollard JW: Macrophages: obligate partners for tumor cell migration, invasion, and metastasis. Cell 2006, 124:263-266

25. Lewis CE, Pollard JW: Distinct role of macrophages in different tumor microenvironments. Cancer Res 2006, 66:605-612

26. Houghton AM: The paradox of tumor-associated neutrophils: fueling tumor growth with cytotoxic substances. Cell Cycle 2010, 9:1732-1737

27. Sinnamon MJ, Carter KJ, Fingleton B, Matrisian LM: Matrix metalloproteinase- 9 contributes to intestinal tumourigenesis in the adenomatous polyposis coli multiple intestinal neoplasia mouse. Int $\mathrm{J}$ Exp Pathol 2008, 89:466-475

28. Belotti D, Paganoni P, Manenti L, Garofalo A, Marchini S, Taraboletti G, Giavazzi R: Matrix metalloproteinases (MMP9 and MMP2) induce the release of vascular endothelial growth factor (VEGF) by ovarian carcinoma cells: implications for ascites formation. Cancer Res 2003 63:5224-5229

29. Ardi VC, Van den Steen PE, Opdenakker G, Schweighofer B, Deryugina El, Quigley JP: Neutrophil MMP-9 proenzyme, unencumbered by TIMP-1, undergoes efficient activation in vivo and catalytically induces angiogenesis via a basic fibroblast growth factor (FGF-2)/ FGFR-2 pathway. J Biol Chem 2009, 284:25854-25866

30. Ebrahem Q, Chaurasia SS, Vasanji A, Qi JH, Klenotic PA, Cutler A, Asosingh K, Erzurum S, Anand-Apte B: Cross-talk between vascular endothelial growth factor and matrix metalloproteinases in the induction of neovascularization in vivo. Am J Pathol 2010, 176:496-503

31. Ardi VC, Kupriyanova TA, Deryugina EI, Quigley JP: Human neutrophils uniquely release TIMP-free MMP-9 to provide a potent catalytic stimulator of angiogenesis. Proc Natl Acad Sci USA 2007, 104 20262-20267

32. Van den Steen PE, Dubois B, Nelissen I, Rudd PM, Dwek RA, Opdenakker G: Biochemistry and molecular biology of gelatinase $B$ or matrix metalloproteinase-9 (MMP-9). Crit Rev Biochem Mol Biol 2002, 37:375-536

33. Okada Y, Gonoji Y, Naka K, Tomita K, Nakanishi I, Iwata K, Yamashita K, Hayakawa T: Matrix metalloproteinase 9 (92-kDa gelatinase/type IV collagenase) from HT 1080 human fibrosarcoma cells: purification and activation of the precursor and enzymic properties. J Biol Chem 267:21712-21719, 1992
34. Ogata $\mathrm{Y}$, Itoh $\mathrm{Y}$, Nagase $\mathrm{H}$ : Steps involved in activation of the promatrix metalloproteinase 9 (progelatinase B)-tissue inhibitor of metalloproteinases-1 complex by 4-aminophenylmercuric acetate and proteinases. J Biol Chem 1995, 270:18506-18511

35. Deryugina El, Zijlstra A, Partridge JJ, Kupriyanova TA, Madsen MA, Papagiannakopoulos T, Quigley JP: Unexpected effect of matrix metalloproteinase down-regulation on vascular intravasation and metastasis of human fibrosarcoma cells selected in vivo for high rates of dissemination. Cancer Res 2005, 65:10959-10969

36. Conn EM, Botkjaer KA, Kupriyanova TA, Andreasen PA, Deryugina EI, Quigley JP: Comparative analysis of metastasis variants derived from human prostate carcinoma cells. roles in intravasation of VEGFmediated angiogenesis and UPA-mediated invasion. Am J Pathol 2009, 175:1638-1652

37. Deryugina EI, Quigley JP: CHAPTER TWO: Chick embryo chorioallantoic membrane models to quantify angiogenesis induced by inflammatory and tumor cells or purified effector molecules. Methods Enzymol 2008, 444:21-41

38. Jablonska J, Leschner S, Westphal K, Lienenklaus S, Weiss S: Neutrophils responsive to endogenous IFN-beta regulate tumor angiogenesis and growth in a mouse tumor model. J Clin Invest 2010, 120:1151-1164

39. Zijlstra A, Seandel M, Kupriyanova TA, Partridge JJ, Madsen MA, HahnDantona EA, Quigley JP, Deryugina El: Proangiogenic role of neutrophillike inflammatory heterophils during neovascularization induced by growth factors and human tumor cells. Blood 2006, 107:317-327

40. Kobayashi Y: Neutrophil infiltration and chemokines. Crit Rev Immunol 2006, 26:307-316

41. Waugh DJ, Wilson C: The interleukin-8 pathway in cancer. Clin Cancer Res 2008, 14:6735-6741

42. Poh TY, Pease J, Young JR, Bumstead N, Kaiser P: Re-evaluation of chicken CXCR1 determines the true gene structure: cXCLi1 (K60) and CXCLi2 (CAF/interleukin-8) are ligands for this receptor. J Bio Chem 2008, 283:16408-16415

43. Sparmann A, Bar-Sagi D: Ras-induced interleukin-8 expression plays a critical role in tumor growth and angiogenesis. Cancer Cell 2004, 6:447458

44. Martin MD, Carter KJ, Jean-Philippe SR, Chang M, Mobashery S, Thiolloy S, Lynch CC, Matrisian LM, Fingleton B: Effect of ablation or inhibition of stromal matrix metalloproteinase- 9 on lung metastasis in a breast cancer model is dependent on genetic background. Cancer Res 2008, 68:6251-6259

45. Opdenakker G, Van den Steen PE, Dubois B, Nelissen I, Van Coillie E, Masure S, Proost P, Van Damme J: Gelatinase B functions as regulator and effector in leukocyte biology. J Leukoc Biol 2001, 69:851-859

46. Roncucci L, Mora E, Mariani F, Bursi S, Pezzi A, Rossi G, Pedroni M, Luppi D, Santoro L, Monni S, Manenti A, Bertani A, Merighi A, Benatti P, Di Gregorio C, de Leon PM: Myeloperoxidase-positive cell infiltration in colorectal carcinogenesis as indicator of colorectal cancer risk. Cancer Epidemiol Biomarkers Prev 2008, 17:2291-2297

47. Shojaei F, Singh M, Thompson JD, Ferrara N: Role of Bv8 in neutrophil-dependent angiogenesis in a transgenic model of cancer progression. Proc Natl Acad Sci USA 2008, 105:2640-2645

48. Sawanobori Y, Ueha S, Kurachi M, Shimaoka T, Talmadge JE, Abe J, Shono Y, Kitabatake M, Kakimi K, Mukaida N, Matsushima K: Chemokine-mediated rapid turnover of myeloid-derived suppressor cells in tumor-bearing mice. Blood 2008, 111:5457-5466

49. Van den Steen PE, Proost P, Wuyts A, Van Damme J, Opdenakker G: Neutrophil gelatinase B potentiates interleukin- 8 tenfold by aminoterminal processing, whereas it degrades CTAP-III. PF-4, and GRO-alpha and leaves RANTES and MCP-2 intact. Blood 2000, 96:2673-2681

50. Nielsen BS, Timshel S, Kjeldsen L, Sehested M, Pyke C, Borregaard N. Dano K: 92 kDa Type IV collagenase (MMP-9) is expressed in neutrophils and macrophages but not in malignant epithelial cells in human colon cancer. Int J Cancer 1996, 65:57-62

51. Madsen MA, Deryugina EI, Niessen S, Cravatt BF, Quigley JP: Activity-based protein profiling implicates urokinase activation as a key step in human fibrosarcoma intravasation. J Biol Chem 2006, 281 : 15997-16005

52. Blouse GE, Botkjaer KA, Deryugina E, Byszuk AA, Jensen JM, Mortensen KK, Quigley JP, Andreasen PA: A novel mode of intervention with serine protease activity: targeting zymogen activation. J Biol Chem 2009, 284:4647-4657 